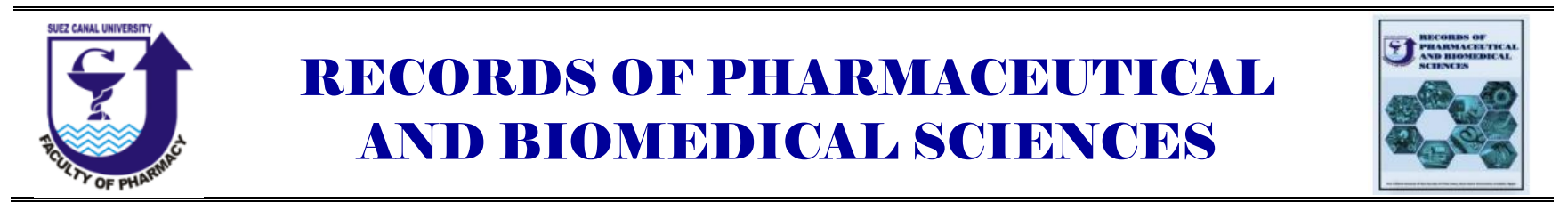

\title{
Genus Holothuria an imminent source of diverse chemical entities: A review
}

\author{
Dina M. Hal ${ }^{a}$, Enas E. Eltamany ${ }^{a}$, Reda F.A. Abdelhameed ${ }^{a}$, Safwat A. Ahmed ${ }^{a}$, Hashim A, Hassanean ${ }^{a}$, \\ Amany K. Ibrahim ${ }^{a^{*}}$ \\ a Department of Pharmacognosy, Faculty of Pharmacy, Suez Canal University, Ismailia, Egypt.
}

Received on: 13. 06. 2020

Revised on: 22. 06. 2020

Accepted on: 26. 06. 2020

Correspondence Author:

Tel: +01091717812

E-mail address:

am kamal66@yahoo.com

\begin{abstract}
Nature is a valuable source for many medicines in use. About 30 per cent of the world's drugs are either natural products or their biologically active derivatives. Natural products display a wide range of chemical structures that even are unapproachable by highly formulated synthetic principles. Compared to the terrestrial, marine organisms have provided the widest diversity; thirty-four of the thirty-six phyla of life exist. One of the most important diverse families of sea cucumbers is the Holothuriidae family, which exist in oceans and shallow waters as well. This family comprises five genera. Among them, genus Holothuria which is the most predominant one and represented in Egypt by eight species. Phytochemical investigation of sea cucumbers belonging to genus Holothuria has led to the isolation and identification of numerous chemical compounds of different classes. The current review demonstrated that genus Holothuria is a rich source of cerebrosides, saponins, fatty acids, amino acids and phenolics.
\end{abstract}

Keywords: Holothuria, Sea cucumber, Secondary metabolites

\section{Introduction:}

Nature is a major source for many medicines in use.

About 30 per cent of the world's drugs are either natural products or their biologically active derivatives. Natural products display a wide range of chemical structures that even are unapproachable by highly formulated synthetic principles. In addition, phytochemicals have introduced novel therapeutic approaches which helped to make new biochemical approaches (Grabley and Sattler, 2003). Compared to the terrestrial ecosystems, marine organisms have provided the widest diversity; thirty-four of the thirty-six phyla of life exist. About 70 per cent of the surface of our planet is occupied by oceans that provide invertebrates with a diverse living climate (Lalli and Parsons, 1993). This incredible biodiversity has provided an amazing chemical library for marine natural products with a wide range of bioactivities (Haefner et al., 2003). Holothuroidea class (sea cucumber) includes nearly 1,400 species of six orders worldwide (Apodida, Aspidochirotida, Elasipodida, Molpadiida, Dendrochirotida, and 
Dactylochirotida) and 25 families (Mohammed $\boldsymbol{e t}$ al., 2016). Sea cucumbers are present in all oceans worldwide; they exist on the ocean floor or near it, and are frequently hidden beneath it. Additionally, they are found in shallow waters as well. Oriental people, mainly the Japanese and Chinese, use sea cucumbers as a source of medicine and food, resulting in large-scale annual harvesting of sea cucumbers, which contributed to the extinction of many species (Ceesay et al., 2012). One of the most important diverse families of sea cucumbers is the Holothuriidae family. This taxon comprises five genera (Honey-Escandón et al., 2015). Among them, genus Holothuria which is the most predominant one according to the database in World Register of Marine Species (http://www.marinespecies.org/) and it is represented in Egypt by eight species (Ahmed $\boldsymbol{e t}$ al., 2016). Phytochemical investigation of Holothuria sea cucumbers has led to the isolation and identification of numerous bioactive phytoconstituents of diverse chemical classes (Bordbar et al., 2011). Based on the aforementioned reports, the aim of this review is to provide a comprehensive update on the chemistry of sea cucumbers belonging to genus Holothuria.

\section{Chemical constituents reported from some species of genus Holothuria:}

2.1: Cerebrosides: Numerous cerebrosides have been isolated in genus Holothuria (Table 1)

Table 1: Cerebrosides reported in genus Holothuria.

\begin{tabular}{|c|c|c|c|}
\hline $\begin{array}{c}\text { Compound } \\
\text { No } \\
\end{array}$ & Species & Compound Name & Reference \\
\hline 1 & H. pervicax & HPG-8 & (Yamada et al., 1998). \\
\hline 2 & H. pervicax & HPG-3 & (Yamada et al., 1998). \\
\hline 3 & H. pervicax & HPG-1 & (Yamada et al., 1998). \\
\hline 4 & H. pervicax & HPG-7 & (Yamada et al., 2000) \\
\hline 5 & H. pervicax & HPC-1 & (Yamada et al., 2002) \\
\hline 6 & H. pervicax & HPC-2 & (Yamada et al., 2002) \\
\hline 7 & H. pervicax & HPC-3-A and HPC-3-B & (Yamada et al., 2002) \\
\hline 8 & H. pervicax & HPC-3-C - HPC-3-J & (Yamada et al., 2002) \\
\hline 9 & H. Leucospilota & HLG-1 & (Yamada et al., 2001) \\
\hline 10 & H. Leucospilota & HLG-2 & (Yamada et al., 2001) \\
\hline 11 & H. Leucospilota & HLG-3 & (Yamada et al., 2001) \\
\hline 12 & H. Leucospilota & HLC-2-A & (Yamada et al., 2005) \\
\hline 13 & H. coronopertusa & $\begin{array}{l}\text { Compound-1-a, Compound-1-b, Compound-2-a, Compound-2-b, } \\
\text { Compound-2-c, Compound-3-a, Compound-3-b, Compound-3-c, } \\
\text { Compound-3-d, Compound-4-a, Compound-4-b, Compound-4-c, } \\
\text { Compound-5-a, Compound-5-b, Compound-5-c, Compound-6-a, } \\
\text { Compound-6-b and Compound-6-c }\end{array}$ & (Hue et al., 2001) \\
\hline
\end{tabular}



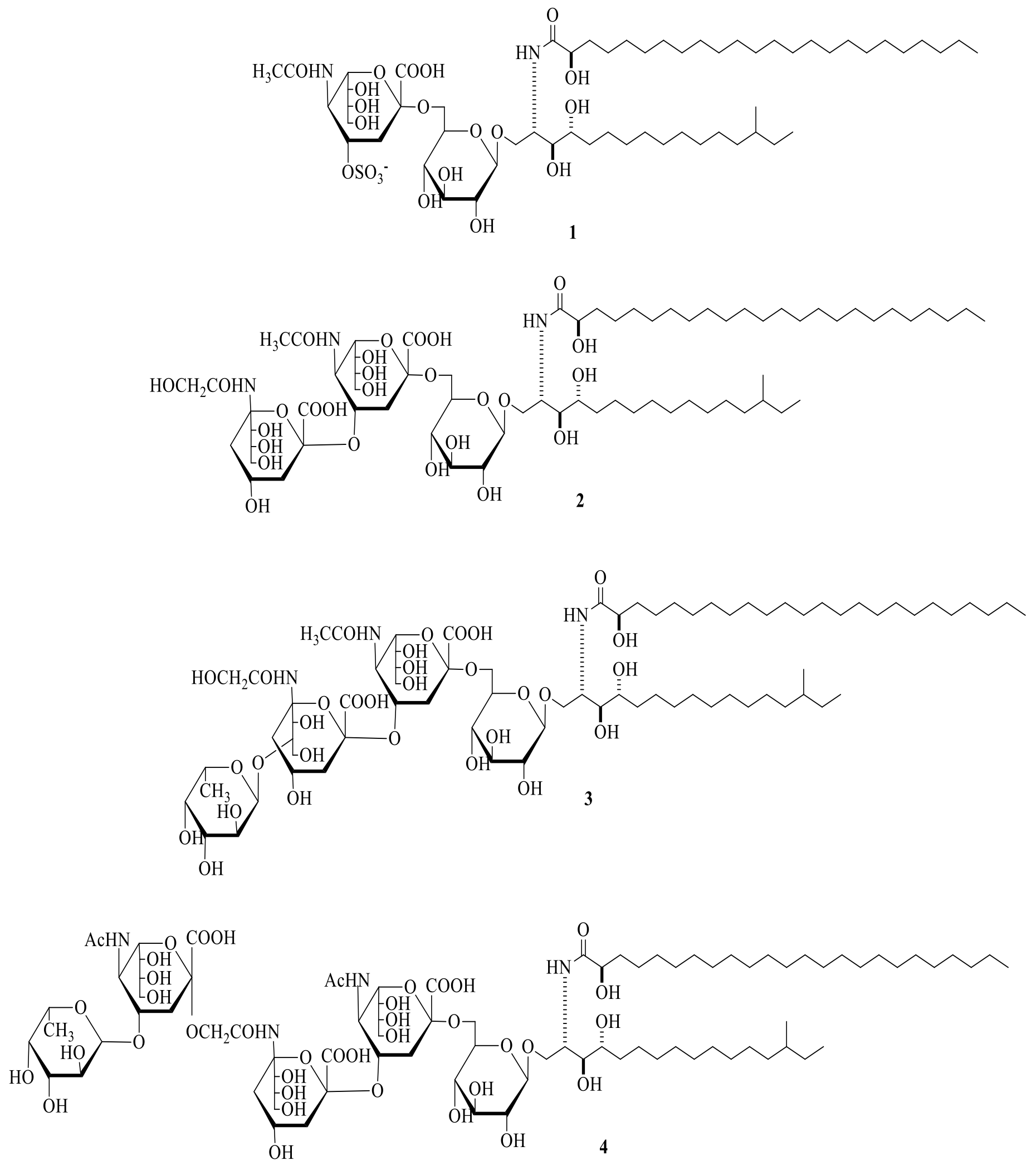

Figure 1: chemical structures of compounds 1-4 

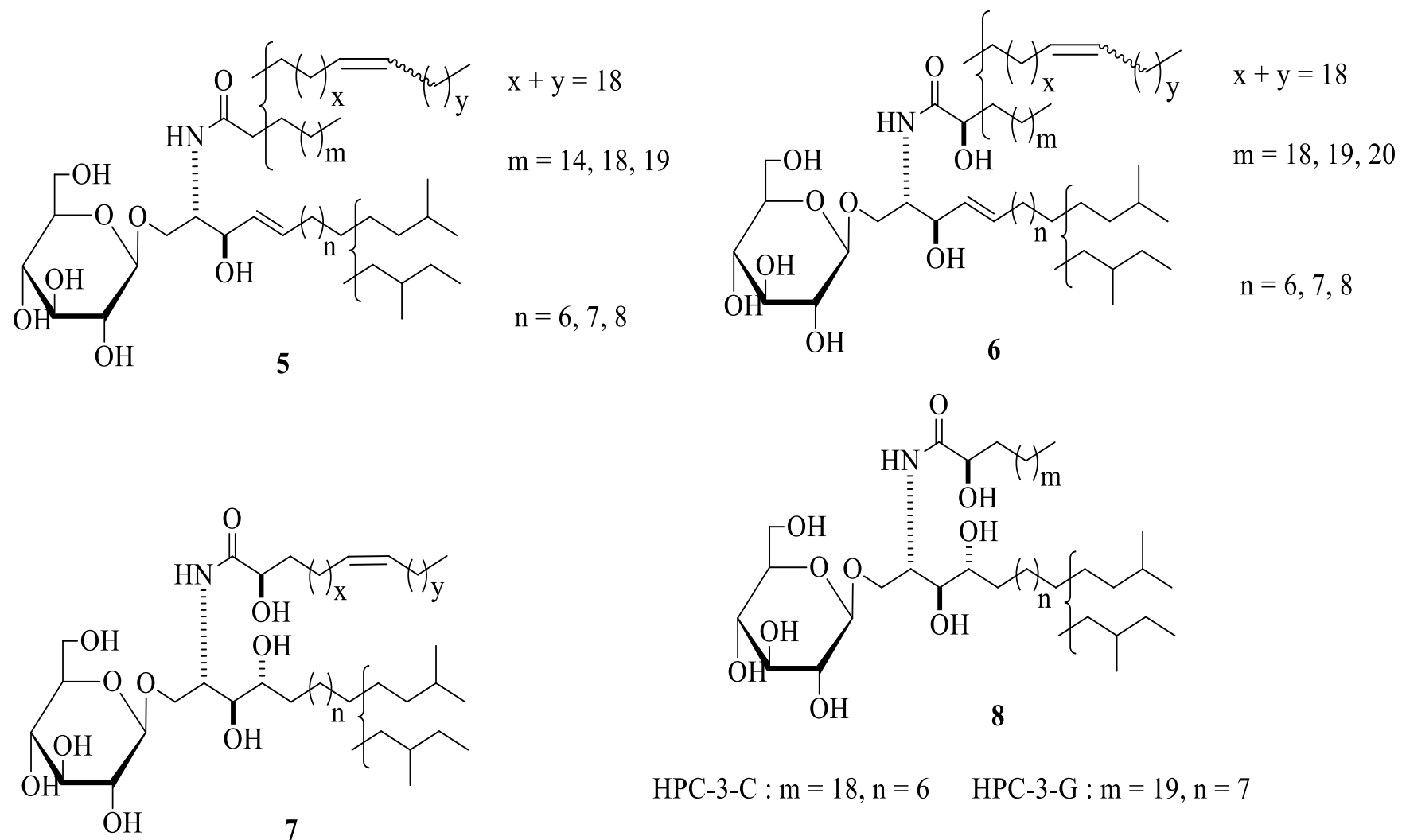

HPC-3-C $: m=18, n=6 \quad$ HPC-3-G $: m=19, n=7$

HPC-3-D : $m=18, \mathrm{n}=7 \quad$ HPC-3-H $: m=20, \mathrm{n}=6$

HPC-3-A : $\mathrm{x}=10, \mathrm{y}=7, \mathrm{n}=6$

HPC-3-E : $m=19, n=6$

HPC-3-I : $\mathrm{m}=19, \mathrm{n}=8$

HPC-3-F : $\mathrm{m}=18, \mathrm{n}=8$

HPC-3-J : $m=20, n=7$

Figure 2: chemical structures of compounds 5-8. 


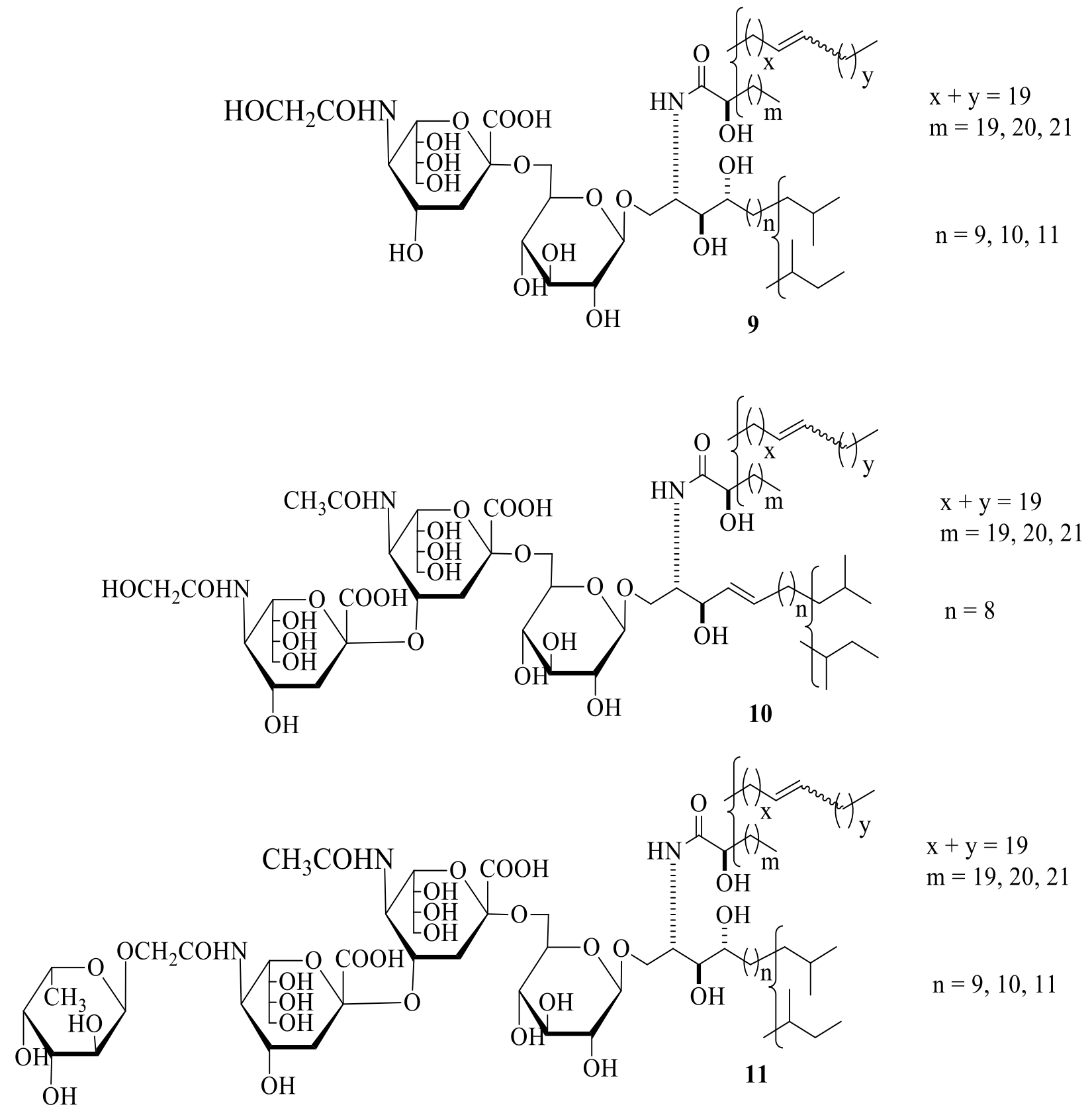

Figure 3: chemical structures of compounds 9-11. 

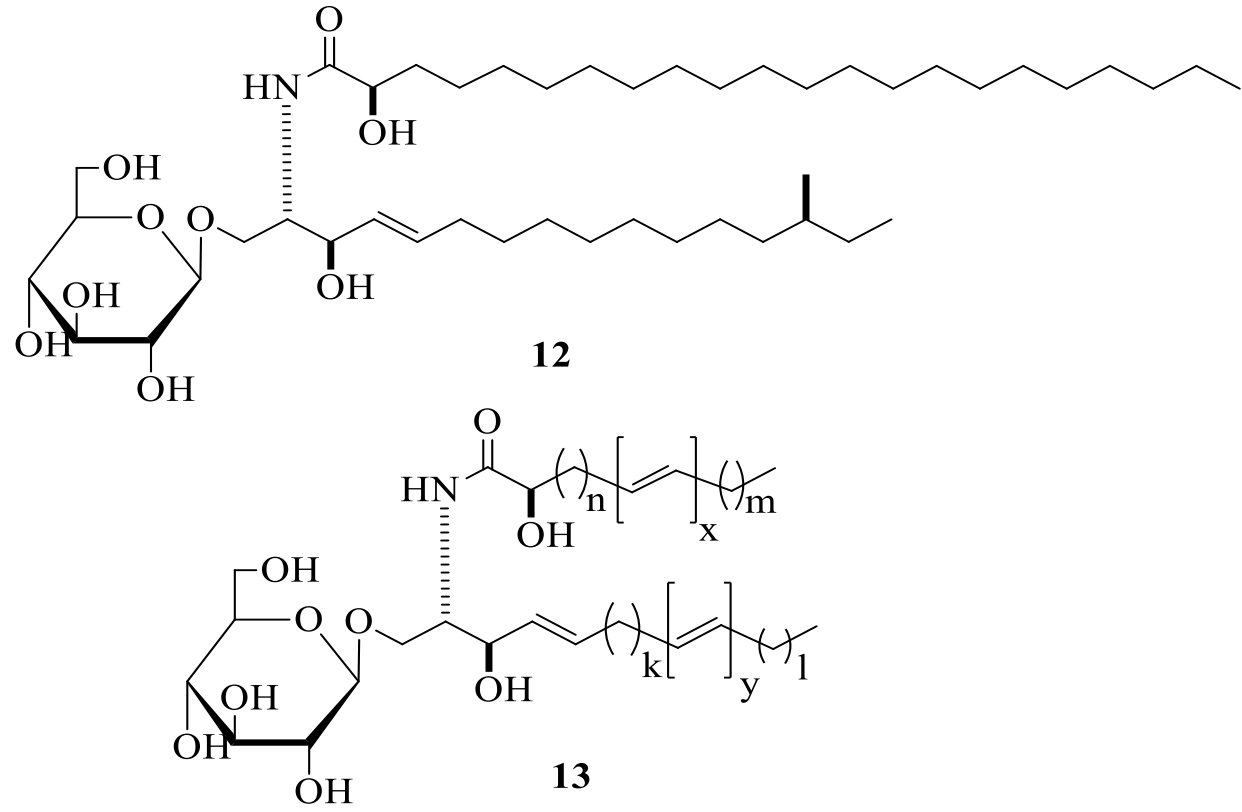

Compound-1-a : $\mathrm{n}=19, \mathrm{x}=0, \mathrm{~m}=0, \mathrm{k}=11, \mathrm{y}=0,1=0$

Compound-1-b : $\mathrm{n}=20, \mathrm{x}=0, \mathrm{~m}=0, \mathrm{k}=10, \mathrm{y}=0,1=0$

Compound-2-a $: \mathrm{n}=19, \mathrm{x}=0, \mathrm{~m}=0, \mathrm{k}=12, \mathrm{y}=0,1=0$

Compound-2-b : $\mathrm{n}=20, \mathrm{x}=0, \mathrm{~m}=0, \mathrm{k}=11, \mathrm{y}=0,1=0$

Compound-2-c : $\mathrm{n}=21, \mathrm{x}=0, \mathrm{~m}=0, \mathrm{k}=10, \mathrm{y}=0,1=0$

Compound-3-a : $\mathrm{n}=19, \mathrm{x}=0, \mathrm{~m}=0, \mathrm{k}=-, \mathrm{y}=1,1=-$

Compound-3-b : $\mathrm{n}=-, \mathrm{x}=1, \mathrm{~m}=-, \mathrm{k}=12, \mathrm{y}=0,1=0$

Compound-3-c : $\mathrm{n}=20, \mathrm{x}=0, \mathrm{~m}=0, \mathrm{k}=-, \mathrm{y}=1,1=-$

Compound-3-d : $\mathrm{n}=12, \mathrm{x}=1, \mathrm{~m}=7, \mathrm{k}=11, \mathrm{y}=0,1=0$

Compound-4-a $: \mathrm{n}=19, \mathrm{x}=0, \mathrm{~m}=0, \mathrm{k}=13, \mathrm{y}=0,1=0$

Compound-4-b : $\mathrm{n}=20, \mathrm{x}=0, \mathrm{~m}=0, \mathrm{k}=12, \mathrm{y}=0,1=0$

Compound-4-c : $\mathrm{n}=21, \mathrm{x}=0, \mathrm{~m}=0, \mathrm{k}=11, \mathrm{y}=0,1=0$

Compound-5-a : $\mathrm{n}=-, \mathrm{x}=1, \mathrm{~m}=-, \mathrm{k}=13, \mathrm{y}=0,1=0$

Compound-5-b : $\mathrm{n}=12, \mathrm{x}=1, \mathrm{~m}=7, \mathrm{k}=12, \mathrm{y}=0,1=0$

Compound-5-c : $\mathrm{n}=-, \mathrm{x}=1, \mathrm{~m}=-, \mathrm{k}=11, \mathrm{y}=0,1=0$

Compound-6-a $: \mathrm{n}=20, \mathrm{x}=0, \mathrm{~m}=0, \mathrm{k}=13, \mathrm{y}=0,1=0$

Compound-6-b : $\mathrm{n}=21, \mathrm{x}=0, \mathrm{~m}=0, \mathrm{k}=12, \mathrm{y}=0,1=0$

Compound-6-c : $\mathrm{n}=22, \mathrm{x}=0, \mathrm{~m}=0, \mathrm{k}=11, \mathrm{y}=0,1=0$

Figure 4: chemical structures of compounds 12 and 13. 


\section{2: Saponins:}

- Sea cucumber triterpene glycosides are categorized into two groups according to the existence or absence of $\gamma(18-20)$ lactone in the glycoside aglycone portion based on their chemical structure. Triterpene glycosides containing $3 \beta$ hydroxy-5 $\alpha$-lanostano- $\gamma$ (18, 20)-lactone are holostane type whereas non holostane type does not contain $\gamma$ (18-20) lactone. Glycosides of the holostane type often vary in their aglycone chemical structure so that they can be further subdivided into three classes:
- Holostane glycosides contain 3 $\beta$-hydroxyholost9(11)-ene. See Tables (2,3,4,5 and 6)

- Holostane glycosides contain 3 $\beta$-hydroxyholost8(9)-ene.

- Holostane glycosides contain 3 $\beta$-hydroxyholost7(8)-ene.

Each of these three groups can also be further divided into additional groups or categories based on the number of sugar units in the holostane glycoside glycone structure (Mondol et al., 2017).

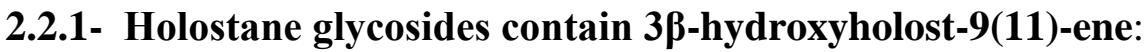

2.2.1.1- Holostane glycosides contain 3及-hydroxyholost-9(11)-ene with 1-3 sugar units oligosaccharide chain:

Table 2: Holostane glycosides contain 3ß-hydroxyholost-9(11)-ene with 1-3 sugar units oligosaccharide chain reported in genus Holothuria:

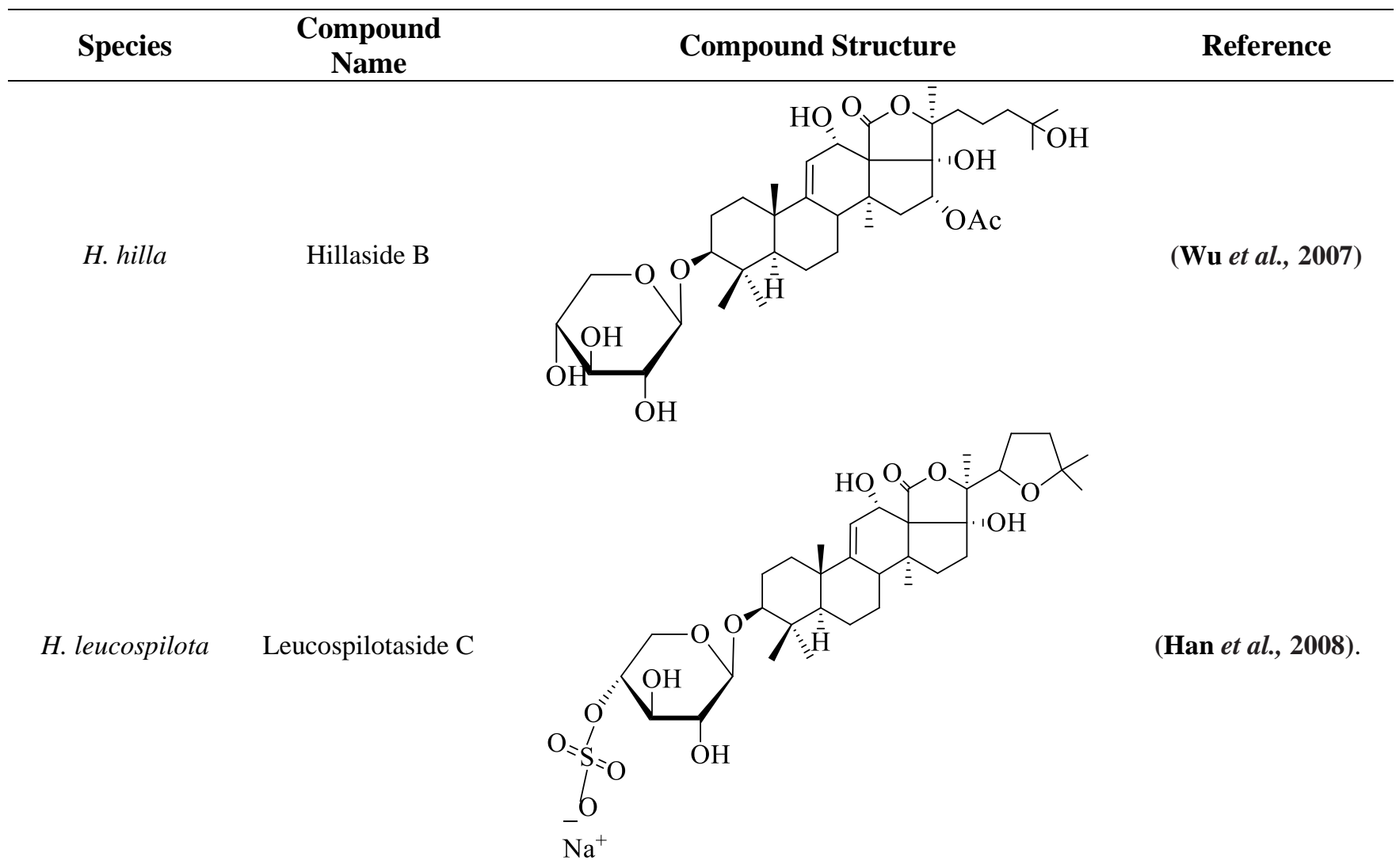


H. nobilis

Nobiliside A

H. nobilis
Nobiliside C

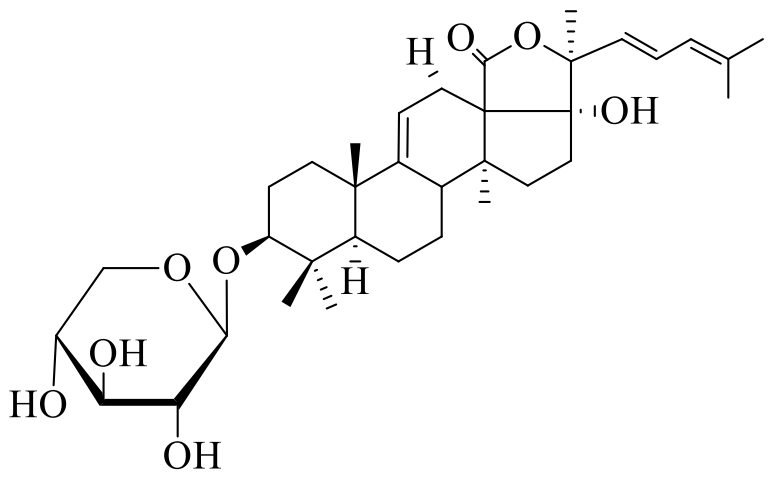

(Wu et al., 2006a)

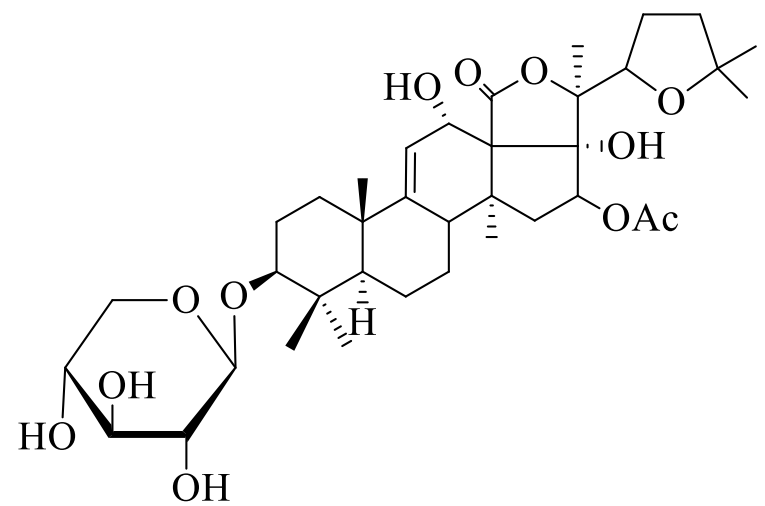

(Wu et al., 2006a)

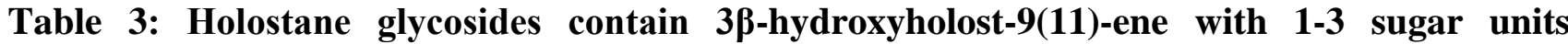
oligosaccharide chain reported in geuns Holothuria:

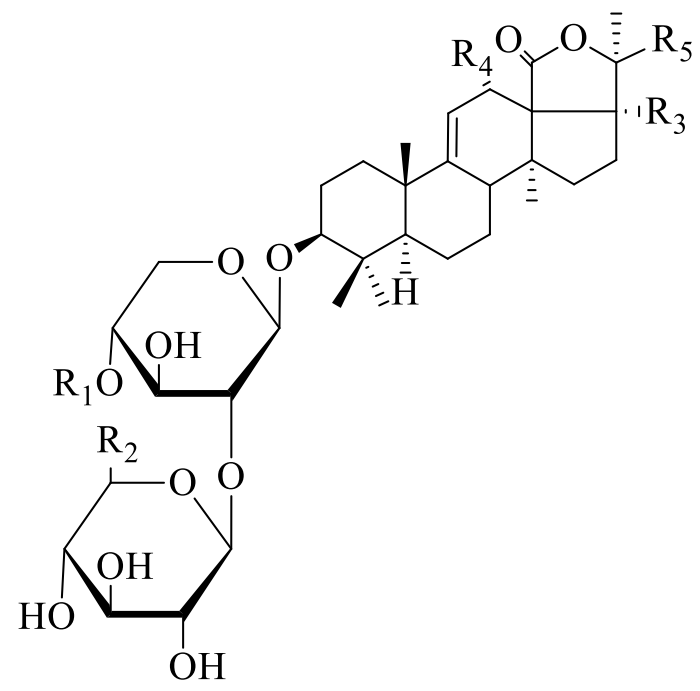

\begin{tabular}{|c|c|c|c|c|c|c|c|}
\hline Species & $\begin{array}{l}\text { Compound } \\
\text { Name }\end{array}$ & $\mathbf{R}^{1}$ & $\mathbf{R}^{2}$ & $\mathbf{R}^{3}$ & $\mathbf{R}^{4}$ & $\mathbf{R}^{5}$ & Reference \\
\hline $\begin{array}{l}\text { H. lubrica, H.arenicola, } \\
\text { H. pervicax, H. nobilis, } \\
\text { H. hilla, H. gracilis, } \\
\text { H. difficilis, H. coluber } \\
\text { H. pulla. H. cubana, }\end{array}$ & Holothurin B & $\mathrm{SO}_{3} \mathrm{Na}$ & $\mathrm{CH}_{3}$ & $\mathrm{OH}$ & $\mathrm{OH}$ & & $\begin{array}{l}\text { (Yasumoto et al., } \\
\text { 1967), } \\
\text { (Elyakov et al., 1973), } \\
\text { (Elyakov } \text { et al., 1975), } \\
\text { (Kitagawa et al., 1979), }\end{array}$ \\
\hline
\end{tabular}


Hal et. al

\begin{tabular}{|c|c|c|c|c|c|c|c|}
\hline $\begin{array}{l}\text { H.grisea, H. surinamensis } \\
\text { H.mexicana, H.atra } \\
\text { H.leucospilota, H.polii, } \\
\text { H.axiloga, H.edulis, } \\
\text { H.tubulosa, H. scabra } \\
\text { and } \\
\text { H. fuscocinerea }\end{array}$ & & & & & & & $\begin{array}{l}\text { (Kobayashi } \text { et } \\
\text { al.,1991), } \\
\text { (Silchenko et al., } \\
\text { 2005), } \\
\text { (Zhang } \text { et al., 2006) } \\
\text { (Dang } \text { et al., 2007) }\end{array}$ \\
\hline H. leucospilota, H. atra & Holothurin $\mathrm{B}_{1}$ & $\mathrm{SO}_{3} \mathrm{Na}$ & $\mathrm{CH}_{3}$ & $\mathrm{OH}$ & $\mathrm{OH}$ & & $\begin{array}{l}\text { (Kitagawa } \text { et al., 1978) } \\
\text { (Kobayashi } \text { et al., } \\
\text { 1991) }\end{array}$ \\
\hline H. polii & Holothurin $\mathrm{B}_{2}$ & $\mathrm{SO}_{3} \mathrm{Na}$ & $\mathrm{CH}_{3}$ & $\mathrm{OH}$ & $\mathrm{OH}$ & & (Silchenko et al., 2005) \\
\hline H. polii & Holothurin $\mathrm{B}_{3}$ & $\mathrm{SO}_{3} \mathrm{Na}$ & $\mathrm{CH}_{3}$ & $\mathrm{H}$ & $\mathrm{OH}$ & & (Silchenko et al., 2005) \\
\hline H. polii & Holothurin $\mathrm{B}_{4}$ & $\mathrm{SO}_{3} \mathrm{Na}$ & $\mathrm{CH}_{3}$ & $\mathrm{OH}$ & $\mathrm{OH}$ & & (Silchenko et al., 2005) \\
\hline H. leucospilota & leucospilotaside A & $\mathrm{SO}_{3} \mathrm{Na}$ & $\mathrm{CH}_{3}$ & $\mathrm{OH}$ & $\mathrm{OH}$ & & (Hua et al., 2009) \\
\hline H. leucospilota & leucospilotaside B & $\mathrm{SO}_{3} \mathrm{Na}$ & $\mathrm{CH}_{3}$ & $\mathrm{OH}$ & $\mathrm{OH}$ & $\mathrm{H}_{\mathrm{OH}}$ & (Hua et al., 2009) \\
\hline H. leucospilota & leucospilotaside D & $\mathrm{SO}_{3} \mathrm{Na}$ & $\mathrm{CH}_{3}$ & $\mathrm{OH}$ & $\mathrm{OH}$ & & $\begin{array}{l}\text { (Honey-Escandón et } \\
\text { al., 2015) }\end{array}$ \\
\hline H. forskali & Holothurinoside D & $\mathrm{H}$ & $\mathrm{CH}_{3}$ & $\mathrm{H}$ & $\mathrm{OH}$ & & $\begin{array}{l}\text { (Rodriguez et al., } \\
\text { 1991) }\end{array}$ \\
\hline H. hilla & Hillaside C & $\mathrm{H}$ & $\mathrm{H}$ & $\mathrm{OH}$ & $\mathrm{OH}$ & $\mathrm{OH}$ & (Wu et al., 2006b). \\
\hline H. nobilis & Nobiliside B & $\mathrm{SO}_{3} \mathrm{Na}$ & $\mathrm{CH}_{2} \mathrm{OH}$ & $\mathrm{OH}$ & $\mathrm{H}$ & & (Wu et al., 2006a) \\
\hline
\end{tabular}

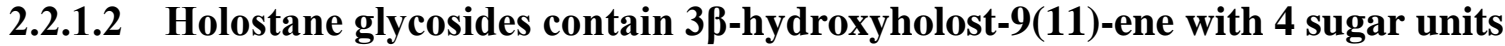
oligosaccharide chain

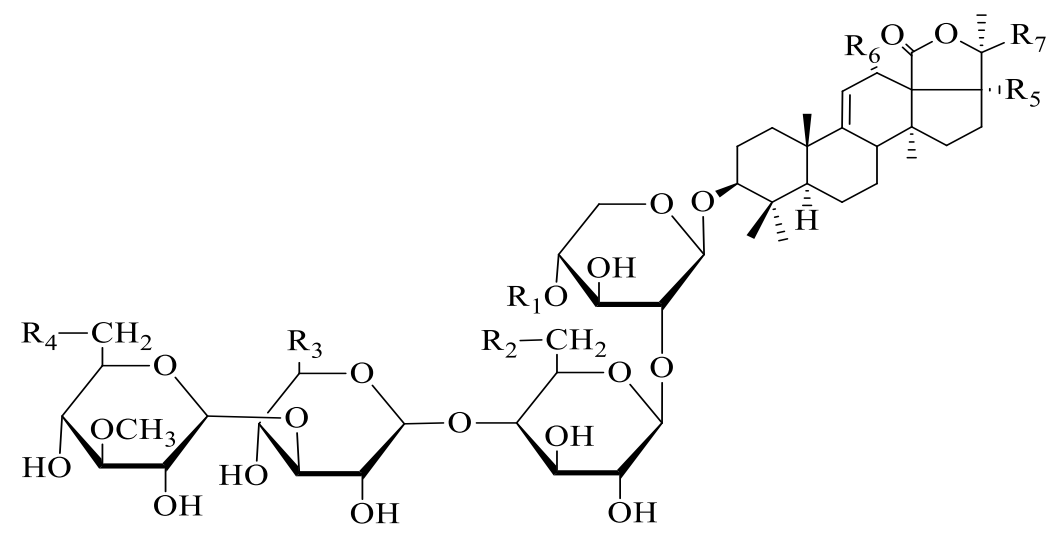


Rec. Pharm. Biomed. Sci. B: 4 (2), 46-67, 2020

Table 4: Holostane glycosides contain 3ß-hydroxyholost-9(11)-ene with 4 sugar units oligosaccharide chain reported in genus Holothuria:

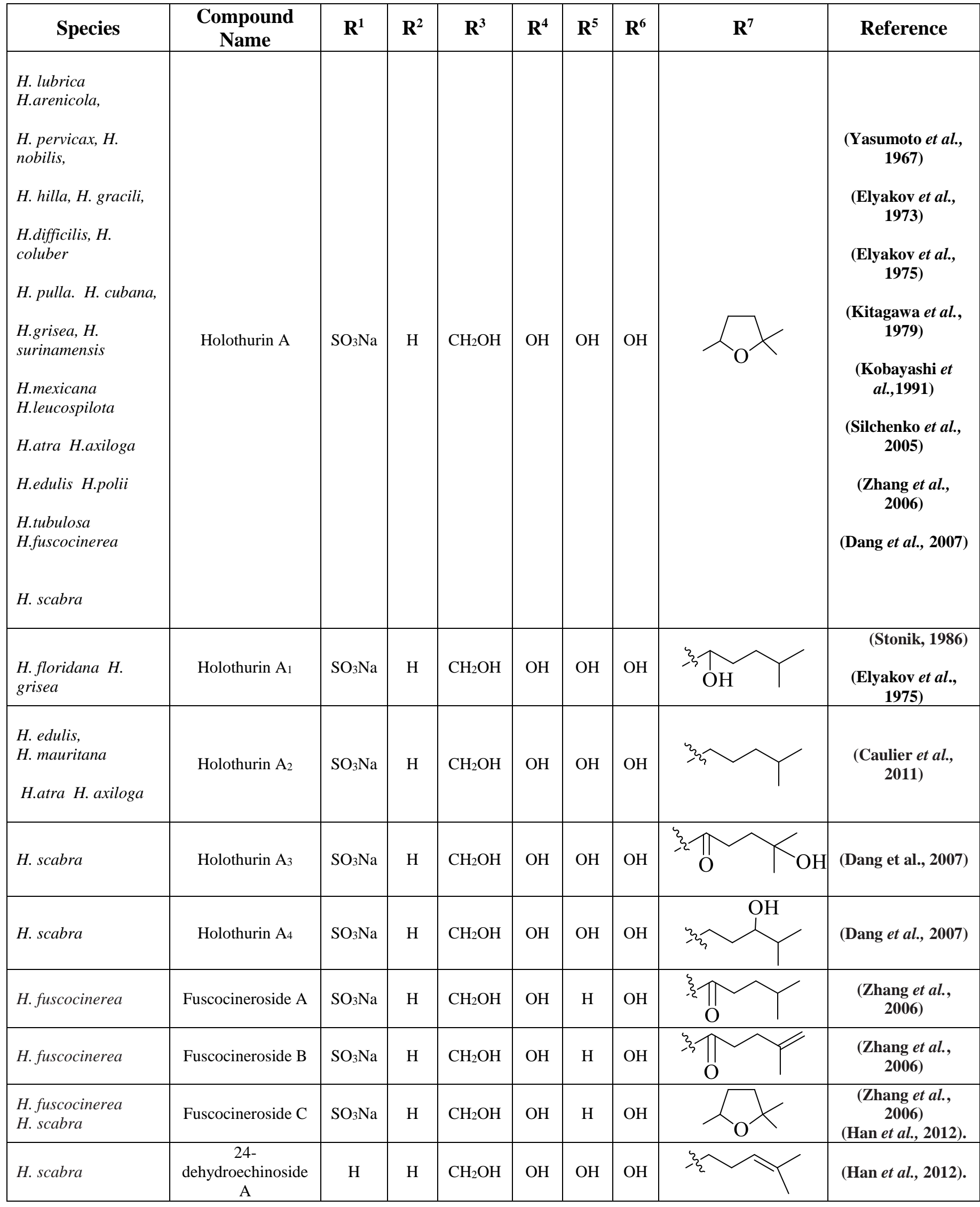




\begin{tabular}{|c|c|c|c|c|c|c|c|c|c|}
\hline H. pervicax & Pervicosides A & $\mathrm{SO}_{3} \mathrm{Na}$ & $\mathrm{H}$ & $\mathrm{CH}_{2} \mathrm{OH}$ & $\mathrm{OH}$ & $\mathrm{H}$ & $\mathrm{OH}$ & $\mathrm{K}_{\mathrm{OAc}}$ & $\begin{array}{l}\text { (Kitagawa et al., } \\
\text { 1989) }\end{array}$ \\
\hline H. pervicax & Pervicosides B & $\mathrm{SO}_{3} \mathrm{Na}$ & $\mathrm{H}$ & $\mathrm{CH}_{2} \mathrm{OH}$ & $\mathrm{OH}$ & $\mathrm{H}$ & $\mathrm{OH}$ & & $\begin{array}{l}\text { (Kitagawa et al., } \\
\text { 1989) }\end{array}$ \\
\hline H. pervicax & Pervicosides C & $\mathrm{SO}_{3} \mathrm{Na}$ & $\mathrm{H}$ & $\mathrm{CH}_{2} \mathrm{OH}$ & $\mathrm{OH}$ & $\mathrm{H}$ & $\mathrm{OH}$ & & $\begin{array}{l}\text { (Kitagawa et al., } \\
\text { 1989) }\end{array}$ \\
\hline H. scabra & Scabraside A & $\mathrm{SO}_{3} \mathrm{Na}$ & $\mathrm{H}$ & $\mathrm{CH}_{2} \mathrm{OH}$ & $\mathrm{OH}$ & $\mathrm{OH}$ & $\mathrm{OH}$ & & (Han et al, 2009). \\
\hline H. scabra & Scabraside B & $\mathrm{SO}_{3} \mathrm{Na}$ & $\mathrm{H}$ & $\mathrm{CH}_{2} \mathrm{OH}$ & $\mathrm{OH}$ & $\mathrm{OH}$ & $\mathrm{OH}$ & & (Han et al, 2009). \\
\hline H. scabra & Scabraside D & $\mathrm{SO}_{3} \mathrm{Na}$ & $\mathrm{H}$ & $\mathrm{CH}_{2} \mathrm{OH}$ & $\mathrm{OH}$ & $\mathrm{OH}$ & $\mathrm{OH}$ & & (Han et al., 2012). \\
\hline H. forskali & Holothurinoside C & $\mathrm{H}$ & $\mathrm{H}$ & $\mathrm{CH}_{2} \mathrm{OH}$ & $\mathrm{OH}$ & $\mathrm{H}$ & $\mathrm{OH}$ & & $\begin{array}{l}\text { (Rodriguez et al., } \\
\text { 1991). }\end{array}$ \\
\hline H. lesson & Holothurinoside J1 & $\mathrm{H}$ & $\mathrm{OH}$ & $\mathrm{CH}_{2} \mathrm{OH}$ & $\mathrm{OH}$ & $\mathrm{OH}$ & $\mathrm{OH}$ & & $\begin{array}{l}\text { (Bahrami } \text { et al., } \\
\text { 2014) }\end{array}$ \\
\hline H. forskali & $\begin{array}{l}\text { Holothurinoside } \\
\text { C1 }\end{array}$ & $\mathrm{H}$ & $\mathrm{OH}$ & $\mathrm{CH}_{2} \mathrm{OH}$ & $\mathrm{OH}$ & $\mathrm{H}$ & $\mathrm{H}$ & & $\begin{array}{l}\text { (Honey-Escandón } \\
\text { et al., 2015) }\end{array}$ \\
\hline H. lesson & Holothurinoside Y & $\mathrm{H}$ & $\mathrm{OH}$ & $\mathrm{CH}_{3}$ & $\mathrm{OH}$ & $\mathrm{OH}$ & $\mathrm{OH}$ & & $\begin{array}{l}\text { (Bahrami et al., } \\
\text { 2014) }\end{array}$ \\
\hline H. lesson & Holothurinoside Z & $\mathrm{H}$ & $\mathrm{OH}$ & $\mathrm{CH}_{3}$ & $\mathrm{OH}$ & $\mathrm{OH}$ & $\mathrm{OH}$ & & $\begin{array}{c}\text { (Bahrami } \text { et al., } \\
\text { 2014) }\end{array}$ \\
\hline H. lesson & Holothurinoside X & $\mathrm{H}$ & $\mathrm{OH}$ & $\mathrm{H}$ & $\mathrm{OH}$ & $\mathrm{OH}$ & $\mathrm{OH}$ & & $\begin{array}{l}\text { (Bahrami } \text { et al., } \\
\text { 2014) }\end{array}$ \\
\hline
\end{tabular}

\subsubsection{Holostane glycosides contain 3及-hydroxyholost-9(11)-ene with 5 sugar units} oligosaccharide chain:

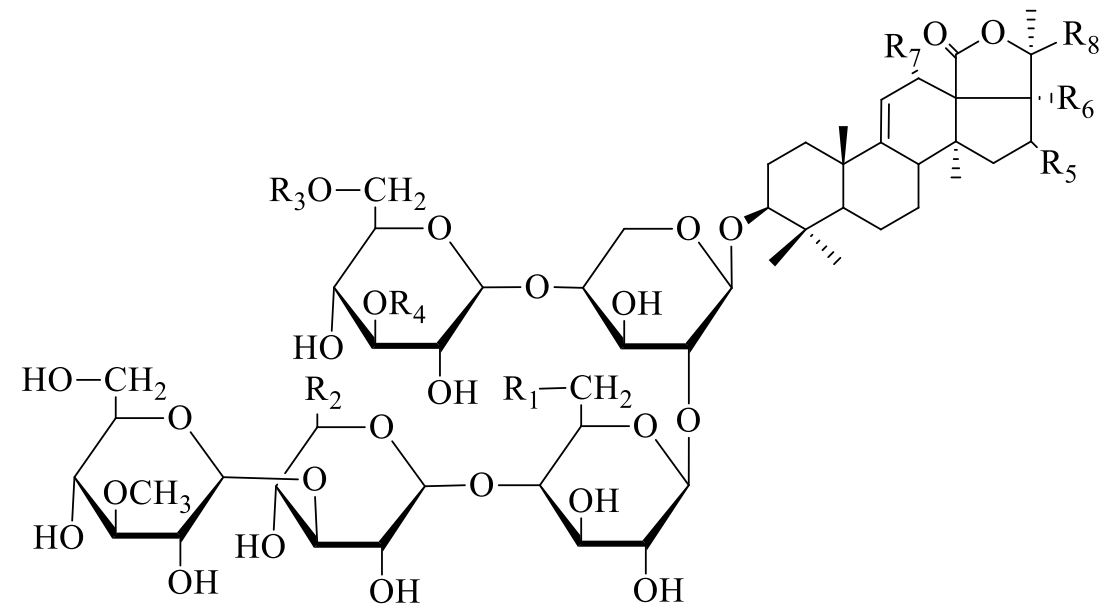


Rec. Pharm. Biomed. Sci. B: 4 (2), 46-67, 2020

Table 5: Holostane glycosides contain 3ß-hydroxyholost-9(11)-ene with 5 sugar units oligosaccharide chain reported in genus Holothuria:

\begin{tabular}{|c|c|c|c|c|c|c|c|c|c|c|}
\hline Species & $\begin{array}{c}\text { Compound } \\
\text { Name }\end{array}$ & $\mathbf{R}^{1}$ & $\mathbf{R}^{2}$ & $\mathbf{R}^{3}$ & $\mathbf{R}^{4}$ & $\mathbf{R}^{5}$ & $\mathbf{R}^{6}$ & $\mathbf{R}^{7}$ & $\mathbf{R}^{8}$ & Reference \\
\hline Hforskalli & Holothurinoside A & $\mathrm{H}$ & $\mathrm{CH}_{2} \mathrm{OH}$ & $\mathrm{H}$ & $\mathrm{H}$ & $\mathrm{H}$ & $\mathrm{OH}$ & $\mathrm{OH}$ & & $\begin{array}{c}\text { (Rodriguez et al., } \\
\text { 1991) }\end{array}$ \\
\hline $\begin{array}{l}\text { H. lesson } \\
\text { H. forskali }\end{array}$ & Holothurinoside $A_{1}$ & $\mathrm{OH}$ & $\mathrm{CH}_{2} \mathrm{OH}$ & $\mathrm{H}$ & $\mathrm{H}$ & $\mathrm{H}$ & $\mathrm{H}$ & $\mathrm{OH}$ & & $\begin{array}{l}\text { (Bahrami } \text { et al., } \\
\text { 2014) } \\
\text { (Rodriguez et al., } \\
\text { 1991) }\end{array}$ \\
\hline H. lesson & Holothurinoside E & $\mathrm{H}$ & $\mathrm{CH}_{2} \mathrm{OH}$ & $\mathrm{H}$ & $\mathrm{H}$ & $\mathrm{H}$ & $\mathrm{H}$ & $\mathrm{OH}$ & & $\begin{array}{c}\text { (Bahrami et al. } \\
\text { 2014) }\end{array}$ \\
\hline H. lesson & Holothurinoside E1 & $\mathrm{OH}$ & $\mathrm{CH}_{2} \mathrm{OH}$ & $\mathrm{H}$ & $\mathrm{H}$ & $\mathrm{H}$ & $\mathrm{H}$ & $\mathrm{H}$ & & $\begin{array}{c}\text { (Bahrami et al., } \\
\text { 2014) }\end{array}$ \\
\hline H. lesson & Holothurinoside M & $\mathrm{H}$ & $\mathrm{CH}_{2} \mathrm{OH}$ & $\mathrm{H}$ & $\mathrm{CH}_{3}$ & $\mathrm{H}$ & $\mathrm{H}$ & $\mathrm{OH}$ & & $\begin{array}{c}\text { (Bahrami et al., } \\
\text { 2014) }\end{array}$ \\
\hline $\begin{array}{c}H . \\
\text { sanctori }\end{array}$ & Holothurinoside $\mathrm{M}_{1}$ & $\mathrm{OH}$ & $\mathrm{CH}_{2} \mathrm{OH}$ & $\mathrm{H}$ & $\mathrm{CH}_{3}$ & $\mathrm{H}$ & $\mathrm{H}$ & $\mathrm{H}$ & & $\begin{array}{c}\text { (Caulier } \text { et al., } \\
\text { 2016) }\end{array}$ \\
\hline H. forskali & $\begin{array}{l}\text { Holothurinoside } \mathrm{N} \\
\text { (Holothurinoside L) }\end{array}$ & $\mathrm{H}$ & $\mathrm{CH}_{2} \mathrm{OH}$ & $\mathrm{H}$ & $\mathrm{CH}_{3}$ & $\mathrm{H}$ & $\mathrm{OH}$ & $\mathrm{OH}$ & & $\begin{array}{l}\text { (Honey- } \\
\text { Escandón et al., } \\
\text { 2015) } \\
\end{array}$ \\
\hline $\begin{array}{c}H . \\
\text { sanctori }\end{array}$ & Holothurinoside $\mathrm{N}_{1}$ & $\mathrm{OH}$ & $\mathrm{CH}_{2} \mathrm{OH}$ & $\mathrm{H}$ & $\mathrm{CH}_{3}$ & $\mathrm{H}$ & $\mathrm{H}$ & $\mathrm{OH}$ & & $\begin{array}{c}\text { (Caulier et al., } \\
\text { 2016) }\end{array}$ \\
\hline $\begin{array}{c}H . \\
\text { forskalli }\end{array}$ & Holothurinoside B & $\mathrm{H}$ & $\mathrm{CH}_{2} \mathrm{OH}$ & $\mathrm{H}$ & $\mathrm{H}$ & $\mathrm{H}$ & $\mathrm{OH}$ & $\mathrm{OH}$ & & $\begin{array}{c}\text { (Rodriguez } \text { et al., } \\
\text { 1991) }\end{array}$ \\
\hline H. grisea & $\begin{array}{c}17- \\
\text { dehydroxyholothurinoside } \\
\text { A } \\
\end{array}$ & $\mathrm{H}$ & $\mathrm{CH}_{2} \mathrm{OH}$ & $\mathrm{H}$ & $\mathrm{CH}_{3}$ & $\mathrm{H}$ & $\mathrm{H}$ & $\mathrm{OH}$ & & $\begin{array}{l}\text { (Sun et al., } \\
\text { 2008). }\end{array}$ \\
\hline H. grisea & Griseaside A & $\mathrm{H}$ & $\mathrm{CH}_{2} \mathrm{OH}$ & $\mathrm{H}$ & $\mathrm{H}$ & $\mathrm{H}$ & $\mathrm{H}$ & $\mathrm{OH}$ & & $\begin{array}{l}\text { (Sun et al., } \\
\text { 2008). }\end{array}$ \\
\hline H. axiloga & Impatienside B & $\mathrm{H}$ & $\mathrm{CH}_{2} \mathrm{OH}$ & $\mathrm{H}$ & $\mathrm{H}$ & $\mathrm{H}$ & $\mathrm{H}$ & $\mathrm{OH}$ & & $\begin{array}{c}\text { (Yuan et al., } \\
\text { 2009) }\end{array}$ \\
\hline H. axiloga & Arguside F & $\mathrm{H}$ & $\mathrm{CH}_{2} \mathrm{OH}$ & $\mathrm{H}$ & $\mathrm{H}$ & OAc & $\mathrm{H}$ & $\mathrm{OH}$ & & $\begin{array}{l}\text { (Yuan et al., } \\
\text { 2009) }\end{array}$ \\
\hline H. axiloga & Pervicoside D & $\mathrm{H}$ & $\mathrm{CH}_{2} \mathrm{OH}$ & $\mathrm{H}$ & $\mathrm{H}$ & $\mathrm{H}$ & $\mathrm{H}$ & $\mathrm{OH}$ & & $\begin{array}{c}\text { (Yuan et al., } \\
\text { 2009) }\end{array}$ \\
\hline
\end{tabular}

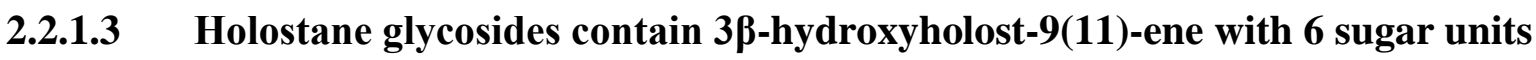
oligosaccharide chain: 
Hal et. al

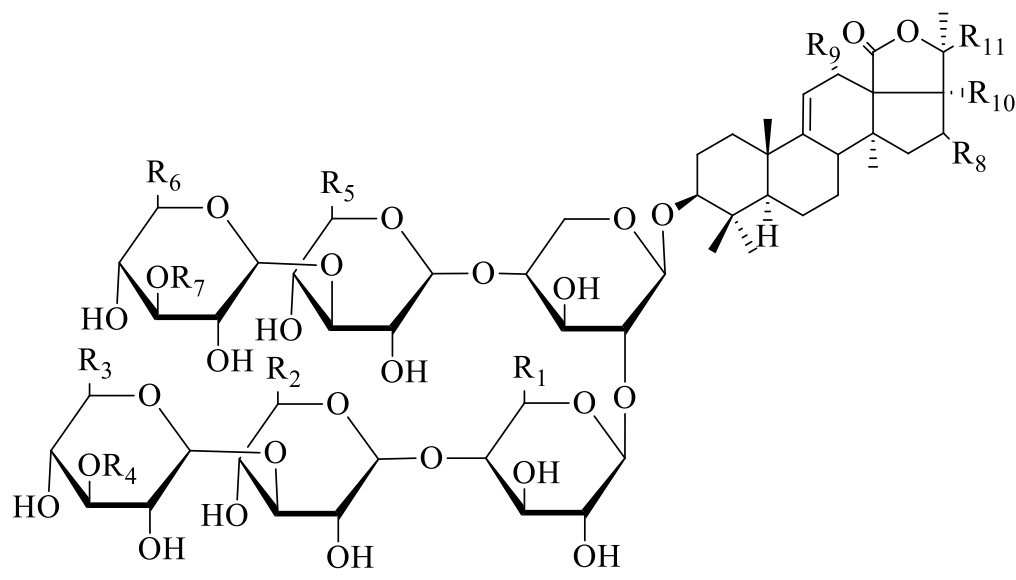

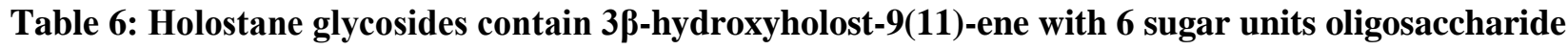
chain reported in genus Holothuria:

\begin{tabular}{|c|c|c|c|c|c|c|c|c|c|c|c|c|c|}
\hline Species & $\begin{array}{l}\text { Compound } \\
\text { Name } \\
\end{array}$ & $\mathbf{R}^{1}$ & $\mathbf{R}^{2}$ & $\mathbf{R}^{3}$ & $\mathbf{R}^{4}$ & $\mathbf{R}^{5}$ & $\mathbf{R}^{6}$ & $\mathbf{R}^{7}$ & $\mathbf{R}^{8}$ & $\mathbf{R}^{9}$ & $\mathbf{R}^{10}$ & $\mathbf{R}^{11}$ & Ref \\
\hline $\begin{array}{c}H . \\
\text { lessoni }\end{array}$ & Lessonioside A & $\mathrm{CH}_{3}$ & $\mathrm{H}$ & $\mathrm{CH}_{2} \mathrm{OH}$ & $\mathrm{CH}_{3}$ & $\mathrm{CH}_{2} \mathrm{OH}$ & $\mathrm{CH}_{2} \mathrm{OH}$ & $\mathrm{CH}_{3}$ & OAc & $\mathrm{OH}$ & $\mathrm{OH}$ & & $\begin{array}{c}\text { (Bahrami } \\
\text { and } \\
\text { Franco, } \\
\text { 2015) }\end{array}$ \\
\hline $\begin{array}{c}H . \\
\text { lessoni }\end{array}$ & Lessonioside B & $\mathrm{CH}_{3}$ & $\mathrm{CH}_{2} \mathrm{OH}$ & $\mathrm{CH}_{3}$ & $\mathrm{H}$ & $\mathrm{CH}_{2} \mathrm{OH}$ & $\mathrm{CH}_{2} \mathrm{OH}$ & $\mathrm{CH}_{3}$ & OAc & $\mathrm{OH}$ & $\mathrm{OH}$ & & $\begin{array}{c}\text { (Bahrami } \\
\text { and } \\
\text { Franco, } \\
\text { 2015) } \\
\end{array}$ \\
\hline $\begin{array}{c}H . \\
\text { lessoni }\end{array}$ & Lessonioside C & $\mathrm{CH}_{2}-\mathrm{OH}$ & $\mathrm{H}$ & $\mathrm{CH}_{2} \mathrm{OH}$ & $\mathrm{CH}_{3}$ & $\mathrm{H}$ & $\mathrm{H}$ & $\mathrm{CH}_{3}$ & $=\mathrm{O}$ & $\mathrm{OH}$ & $\mathrm{OH}$ & & $\begin{array}{c}\text { (Bahrami } \\
\text { and } \\
\text { Franco, } \\
\text { 2015) }\end{array}$ \\
\hline $\begin{array}{c}H . \\
\text { essoni }\end{array}$ & Lessonioside D & $\mathrm{CH}_{3}$ & $\mathrm{CH}_{2} \mathrm{OH}$ & $\mathrm{H}$ & $\mathrm{CH}_{3}$ & $\mathrm{CH}_{2} \mathrm{OH}$ & $\mathrm{CH}_{2} \mathrm{OH}$ & $\mathrm{CH}_{3}$ & OAc & $\mathrm{OH}$ & $\mathrm{OH}$ & & $\begin{array}{c}\text { (Bahrami } \\
\text { and } \\
\text { Franco, } \\
\text { 2015) }\end{array}$ \\
\hline $\begin{array}{c}H . \\
\text { lessoni }\end{array}$ & Lessonioside E & $\mathrm{CH}_{3}$ & $\mathrm{CH}_{2} \mathrm{OH}$ & $\mathrm{CH}_{2} \mathrm{OH}$ & $\mathrm{H}$ & $\mathrm{H}$ & $\mathrm{H}$ & $\mathrm{CH}_{3}$ & $=\mathrm{O}$ & $\mathrm{OH}$ & $\mathrm{OH}$ & & $\begin{array}{c}\text { (Bahrami } \\
\text { and } \\
\text { Franco, } \\
\text { 2015) } \\
\end{array}$ \\
\hline $\begin{array}{c}H . \\
\text { essoni }\end{array}$ & Lessonioside F & $\mathrm{CH}_{3}$ & $\mathrm{CH}_{2} \mathrm{OH}$ & $\mathrm{CH}_{2} \mathrm{OH}$ & $\mathrm{H}$ & $\mathrm{CH}_{2} \mathrm{OH}$ & $\mathrm{CH}_{2} \mathrm{OH}$ & $\mathrm{CH}_{3}$ & $=0$ & $\mathrm{OH}$ & $\mathrm{OH}$ & & $\begin{array}{c}\text { (Bahrami } \\
\text { and } \\
\text { Franco, } \\
\text { 2015) }\end{array}$ \\
\hline $\begin{array}{c}H . \\
\text { lessoni }\end{array}$ & Lessonioside G & $\mathrm{CH}_{2}-\mathrm{OH}$ & $\mathrm{H}$ & $\mathrm{CH}_{2} \mathrm{OH}$ & $\mathrm{CH}_{3}$ & $\mathrm{CH}_{2} \mathrm{OH}$ & $\mathrm{CH}_{2} \mathrm{OH}$ & $\mathrm{CH}_{3}$ & $=0$ & $\mathrm{OH}$ & $\mathrm{OH}$ & & $\begin{array}{c}\text { (Bahrami } \\
\text { and } \\
\text { Franco, } \\
\text { 2015) } \\
\end{array}$ \\
\hline $\begin{array}{c}H . \\
\text { forskali }\end{array}$ & $\begin{array}{l}\text { Holothurinoside } \\
\qquad \mathrm{s} \mathrm{F}_{1}\end{array}$ & $\mathrm{CH}_{2}-\mathrm{OH}$ & $\mathrm{CH}_{2} \mathrm{OH}$ & $\mathrm{CH}_{2} \mathrm{OH}$ & $\mathrm{CH}_{3}$ & $\mathrm{CH}_{2} \mathrm{OH}$ & $\mathrm{CH}_{3}$ & $\mathrm{H}$ & $\mathrm{H}$ & $\mathrm{H}$ & $\mathrm{H}$ & & $\begin{array}{l}\text { (Honey- } \\
\text { Escandón } \\
\text { et al., } \\
2015) \\
\end{array}$ \\
\hline $\begin{array}{c}H . \\
\text { forskali }\end{array}$ & $\begin{array}{l}\text { Holothurinoside } \\
\qquad \mathrm{s} \quad \mathrm{G}_{1}\end{array}$ & $\mathrm{CH}_{2}-\mathrm{OH}$ & $\mathrm{CH}_{2} \mathrm{OH}$ & $\mathrm{CH}_{2} \mathrm{OH}$ & $\mathrm{CH}_{3}$ & $\mathrm{CH}_{2} \mathrm{OH}$ & $\mathrm{CH}_{3}$ & $\mathrm{H}$ & $\mathrm{H}$ & $\mathrm{OH}$ & $\mathrm{H}$ & & $\begin{array}{c}\text { (Honey- } \\
\text { Escandón } \\
\text { et al., } \\
\text { 2015) } \\
\end{array}$ \\
\hline $\begin{array}{c}H . \\
\text { forskali }\end{array}$ & $\begin{array}{l}\text { Holothurinoside } \\
\text { s G }\end{array}$ & $\mathrm{CH}_{3}$ & $\mathrm{CH}_{2} \mathrm{OH}$ & $\mathrm{CH}_{2} \mathrm{OH}$ & $\mathrm{CH}_{3}$ & $\mathrm{CH}_{2} \mathrm{OH}$ & $\mathrm{CH}_{3}$ & $\mathrm{H}$ & $\mathrm{H}$ & $\mathrm{OH}$ & $\mathrm{OH}$ & & $\begin{array}{c}\text { (Honey- } \\
\text { Escandón } \\
\text { et al., } \\
\text { 2015) }\end{array}$ \\
\hline
\end{tabular}




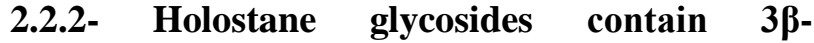
hydroxyholost-8(9)-ene:

- Until now there is no triterpene glycosides with 3ß-hydroxyholost-8(9)-ene isolated from Holothuria genus.

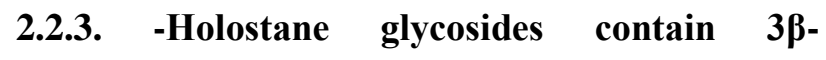
hydroxyholost-7(8)-ene:

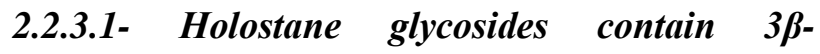
hydroxyholost-7(8)-ene with 1-3 sugar units oligosaccharide chain:

Hillaside $\mathrm{A}$ is a triterpene glycoside with a monosaccharide chain which was isolated from Holothuria hilla sea cucumber, its structure was deduced with spectral data and chemical analysis (Wu et al., 2007).

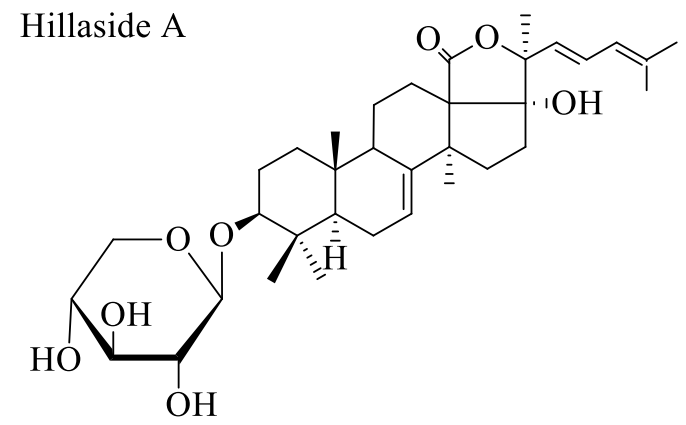

- Holostane glycosides contain $3 \beta$ hydroxyholost-7(8)-ene with 4 sugar units oligosaccharide chain:

Until now there is no triterpene glycosides with 3ß-hydroxyholost-7(8)-ene with 4 sugar units isolated from Holothuria genus.

- Holostane glycosides contain 3及hydroxyholost-7(8)-ene with 5 sugar units oligosaccharide chain:

Up to now there is no triterpene glycosides with $3 \beta$-hydroxyholost-7(8)-ene with 5 sugar units isolated from Holothuria genus.

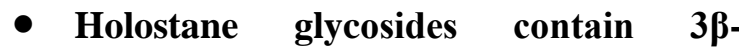
hydroxyholost-7(8)-ene with 6 sugar units oligosaccharide chain:

Up till now there is no triterpene glycosides with 3ß-hydroxyholost-7(8)-ene with 6 sugar units isolated from Holothuria genus.

2.3: Fatty acids: Several fatty acids have been identified in genus Holothuria (Table 7)

Table 7: Fatty acids reported in genus Holothuria

\begin{tabular}{|c|c|c|c|}
\hline \multicolumn{1}{|c|}{ Species } & $\begin{array}{c}\text { Compound } \\
\text { Name }\end{array}$ & Reference \\
\hline $\begin{array}{l}\text { H. Leucospilota } \\
\text { H. sacbra }\end{array}$ & Palmitic acid & (Ceesay et al., 2019) \\
(Yahyavi et al., 2012) \\
$\begin{array}{c}\text { H. Leucospilota } \\
\text { H. Sacbra } \\
\text { H. tubulosa, } \\
\text { H. mammata } \\
\text { H. polii }\end{array}$ & Arachidonic acid & (Yahyavi et al., 2012) \\
(Mercedes et al., 2018).
\end{tabular}


Hal et. al

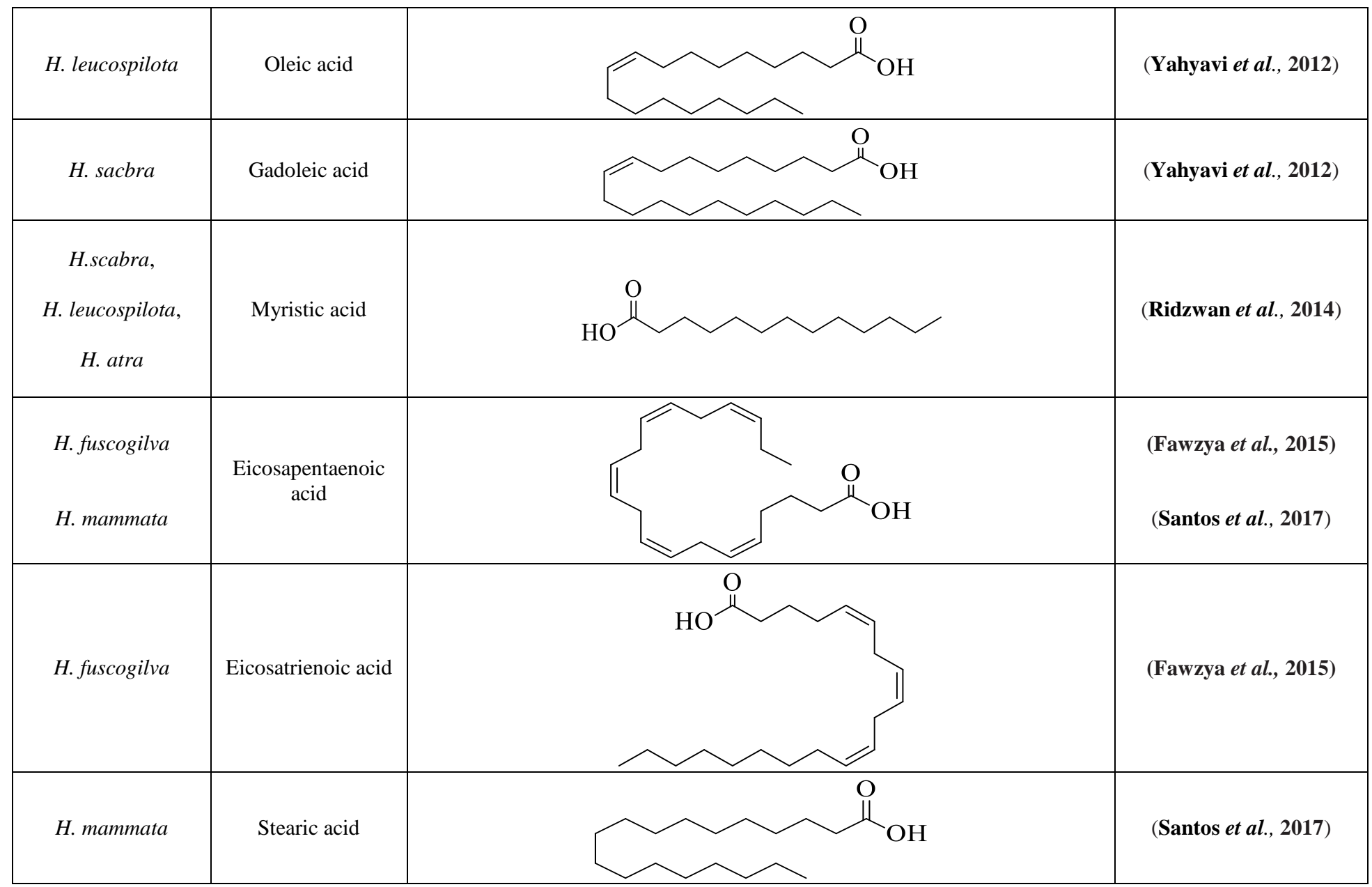

2.4: Amino acids: Numerous amino acids have been reported in genus Holothuria (Table 8)

Table 8: Amino acids reported in genus Holothuria.

\begin{tabular}{|c|c|c|c|}
\hline Species & $\begin{array}{c}\text { Compound } \\
\text { Name }\end{array}$ & Compound Structure & Reference \\
\hline H. scabra & Glycine & (Sroyraya et al., 2017) \\
\hline H. scabra & Proline & (Sroyraya et al., 2017) \\
\hline H. scabra & Glutamic & & (Sroyraya et al., 2017) \\
\hline H. scabra & Alanine & (Ridwanudin et al., 2018) \\
\hline
\end{tabular}


Rec. Pharm. Biomed. Sci. B: 4 (2), 46-67, 2020

\begin{tabular}{|c|c|c|c|}
\hline $\begin{array}{c}\text { H. fuscopunctata, } \\
\text { H. fuscogilva }\end{array}$ & Threonine & (Wen et al., 2010) \\
\hline $\begin{array}{c}\text { H. fuscopunctata, } \\
\text { H. fuscogilva }\end{array}$ & Tyrosine & (Wen et al., 2010) \\
\hline $\begin{array}{c}\text { H. fuscopunctata, } \\
\text { H. fuscogilva }\end{array}$ & Phenylalanine & (Wen et al., 2010) \\
\hline
\end{tabular}

\section{5 : Polysaccharides}

- There are two types of sea cucumber polysaccharides have been isolated fucosylated chondroitin sulfate (FuCS) and fucan sulfates which usually called fucoidans. The fucoidans structure is usually made from linear or branched $(\alpha 1 \rightarrow 3)-$ and/or $(\alpha 1 \rightarrow 4)$-linked fucosyl backbones with sulfate group substitute at C-2 and/or C-4.Fucoidans structures usually differ according to using different extraction procedures and different species so they may differ in monosaccharide composition, different linkages modes, molecular masses and sulfation patterns

(Mansour et al., 2019).

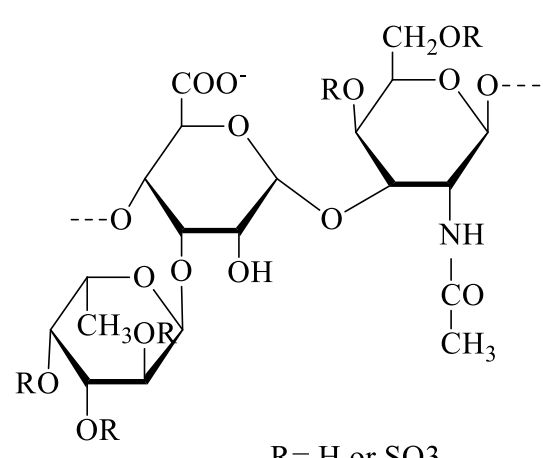

$$
\begin{aligned}
& \mathrm{R}=\mathrm{H} \text { or } \mathrm{SO} 3 \\
& \text { Fucosylated chondroitin sulfate } \\
& \text { FuCS preponderant structure }
\end{aligned}
$$

- Neutral glycans can be considered the third type of sea cucumber polysaccharides but it was only isolated from Holothuria edulis species (Luo et al., 2013).

- Fucosylated chondroitin sulfate (FuCS) and sulfated fucan polysaccharides were isolated from Holothuria nobilis and Holothuria edulis, The FuCSs showed higher anticoagulant activity than the sulfated fucans, despite of the fact that the FuCSs molecular sizes are smaller than the sulfated fucans polysaccharides (Santos $\boldsymbol{e t}$ al., 2017).

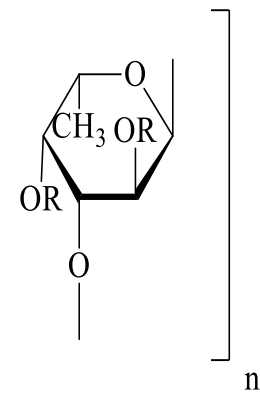

Basic backbone of 1-3-linked fucose polysaccharide in most sulfated fucans in sea cucumbers. R may be $\mathrm{H}, \mathrm{SO}_{3}$, or a fucose or galactose side chain. 


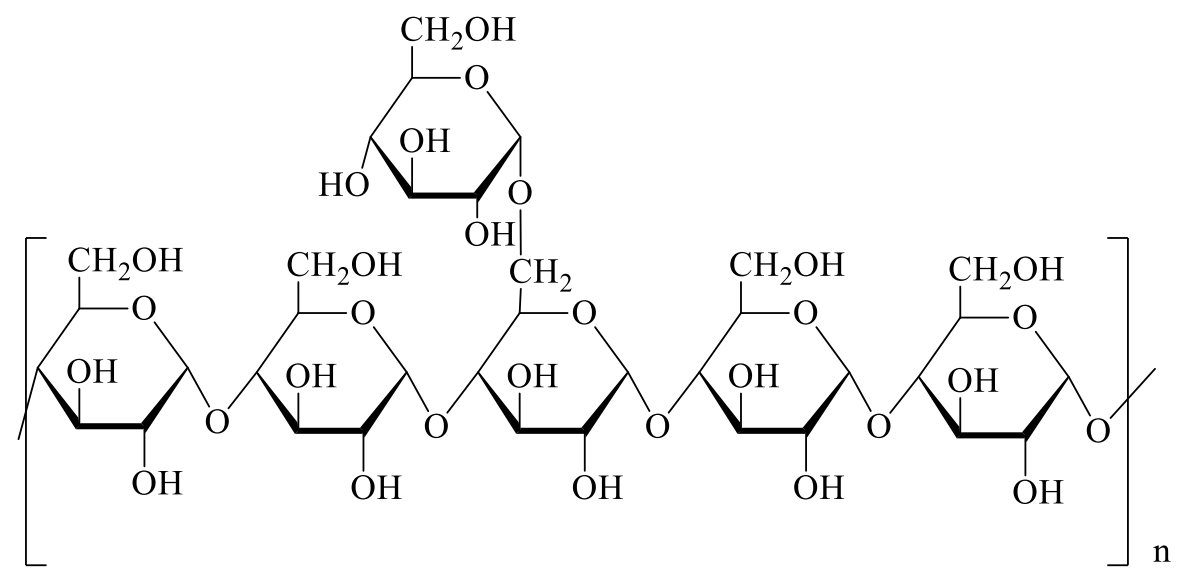

Holothuria edulis neutral glucan

The main residue of polysaccharide is an alpha-(1-4)-D-glucan branched with a single alpa-D-glucose at C-6 every five residues on average

- Two sulfated fucans were isolated from Ludwigothurea grisea (Holothuria grisea) and Holothuria edulis sea cucumbers and their structures were illustrated by the combination of NMR spectroscopy, chemical analysis, methylation experiments and specific optical rotation. The two sulfated $\alpha$-L-fucans are linear polysaccharides which consist of a

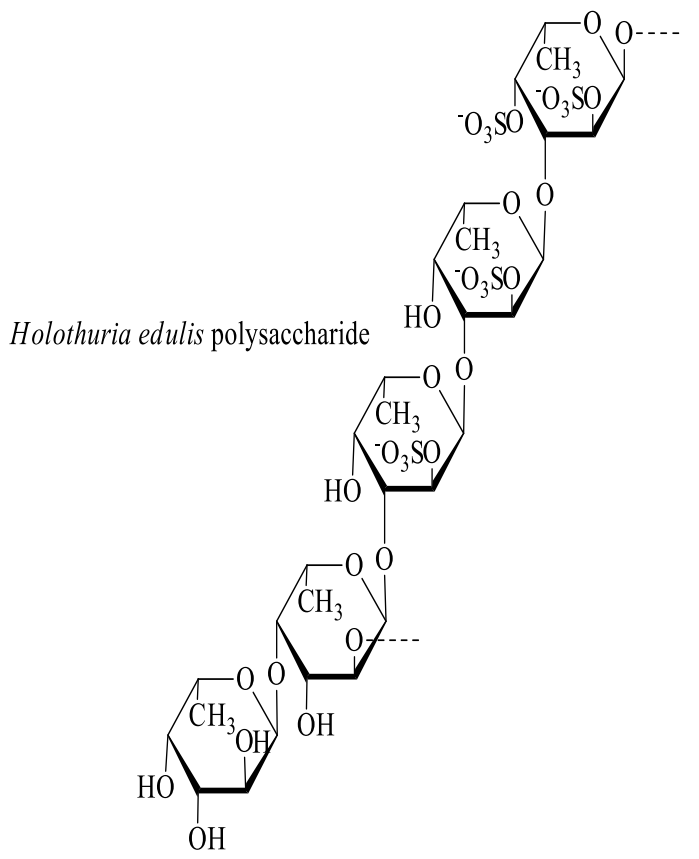

repeated tetrasaccharide backbone unit characterized by O-sulfation pattern with an unsulfated side chain fucose unit. All $H$. edulis $\alpha$ L-fucan polysaccharides are fucosylated at position O-4 of the tetrasaccharide repeated unit but the L.grisea sulfated fucans are not all fucosylated at 4position, only $40-50 \%$ are fucosylated at the backbone (Wu et al., 2015).

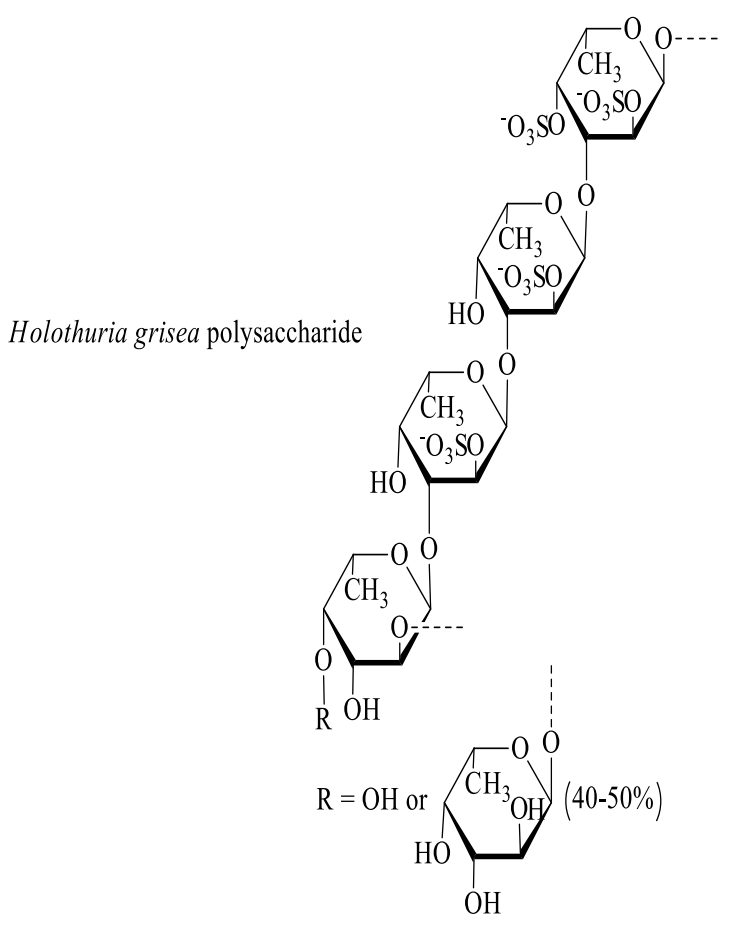


Rec. Pharm. Biomed. Sci. B: 4 (2), 46-67, 2020

2.6: Miscellaneous compounds:

Table 9: Miscellaneous compounds reported in genus Holothuria.

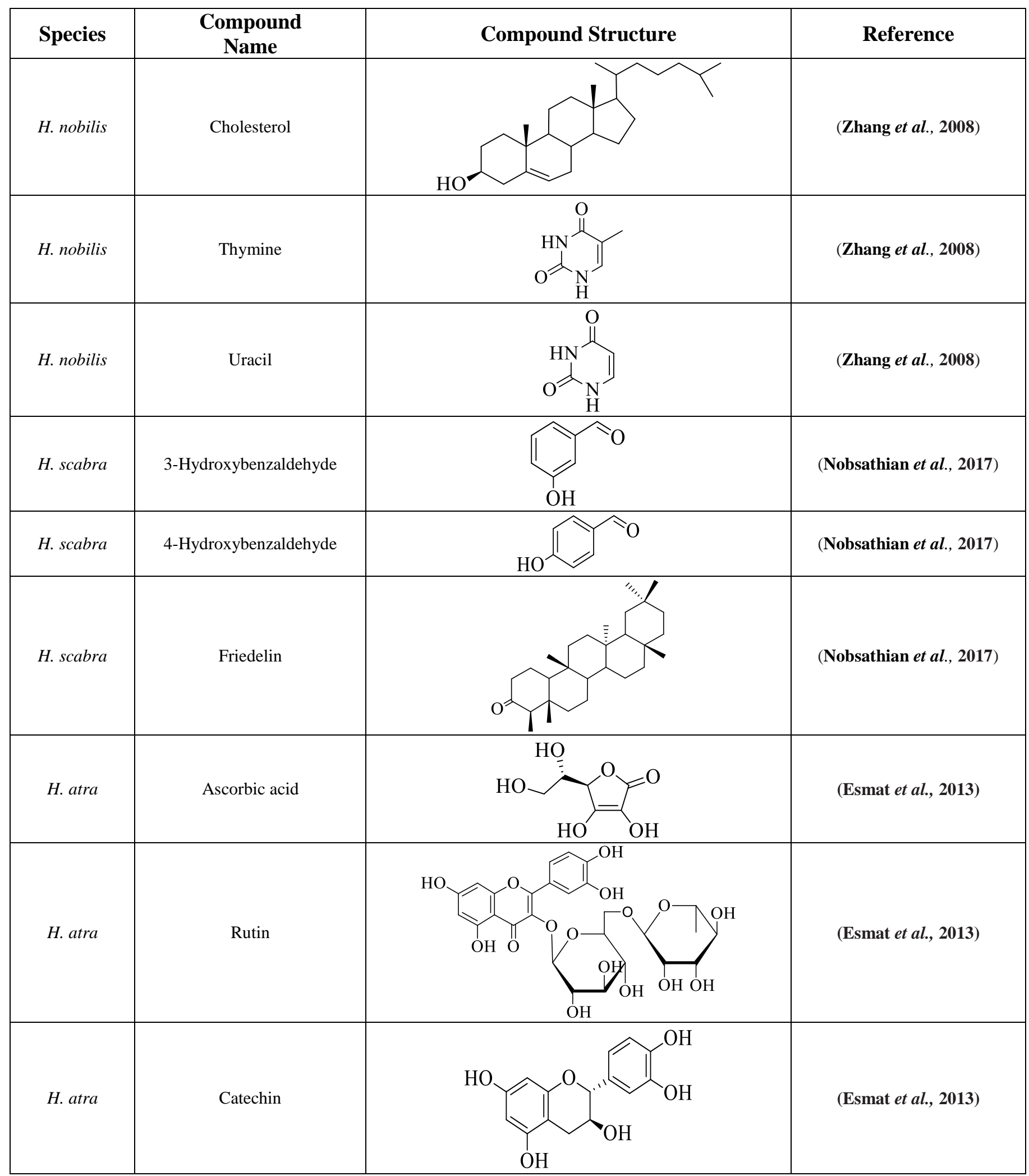




\begin{tabular}{|c|c|c|c|}
\hline H. atra & Chlorogenic acid & (Esmat et al., 2013) \\
\hline H. atra & Coumaric acid & (Esmat et al., 2013) \\
\hline H. atra & Pyrogallol & (Esmat et al., 2013) \\
\hline
\end{tabular}

\section{Conclusion:}

In this review we covered comprehensively the phytochemicals reported in sea cucumbers belonging to genus Holothuria. Our study revealed that this genus of sea cucumber is a precious source of chemically different natural products, especially cerebrosides, saponins, and fatty acids.

\section{References:}

Ahmed, M.I., Aamer, M.A., Lawrence, A.J. 2016. Identification of the Holothurian species of the Red Sea and Gulf of Aqaba using DNA barcoding technique. Egyptian Journal of Aquatic Biology and Fisheries. 20, 1-7. 10.21608/ejabf.2016.11127.

Bahrami, Y., Zhang, W., Chataway, T., Franco, C. 2014. Structural elucidation of novel saponins in the sea cucumber Holothuria lessoni. Marine Drugs, 12, 4439-4473.

Bahrami, Y. and Franco, C. M. M. 2015. Structure elucidation of new acetylated saponins, lessoniosides A, B, C, D, and E, and non-acetylated saponins, lessoniosides $\mathrm{F}$ and $\mathrm{G}$ from the viscera of the sea cucumber Holothuria lessoni. Marine Drugs, 13, 597-617.

Bordbar, S., Anwar, F., Saari, N. 2011. High-Value components and bioactives from sea cucumbers for functional foods - A Review. Marine Drugs, 9, 1761-1805.
Caulier, G., Dyck, S. V., Gerbaux, P., Eeckhaut, I., Flammang, P. 2011. Review of saponin diversity in sea cucumbers belonging to the family Holothuriidae. Beche-de-Mer Information. Bulletin, 31, 48-54.

Caulier, G., Mezali, K., Soualili, D., Decroo, C., Demeyer, M., Eeckhaut, I., Gerbaux, P., Flammang, P. 2016. Chemical characterization of saponins contained in the body wall and the Cuvierian tubules of the sea cucumber Holothuria (Platyperona) sanctori (Delle Chiaje, 1823). Biochemical Systemic and Ecology, 68, 119-127.

Ceesay, A., Shamsudin, M. N., Alipiah, N. M., Ismail, I. S. 2012. Biodiversity and Adaptability of Holothuria leucospilota and Stichopus japonicus sea cucumber species in artificial environment. Journal of Aquaculture Research \& Development, 3,123 .

Ceesay, A., Shamsudin, M. N., Aliyu-Paiko, M., Ismail, I. S., Nazarudin, M. F., Alipiah, N. M. 2019. Extraction and Characterization of Organ Components of the Malaysian Sea Cucumber Holothuria leucospilota Yielded Bioactives Exhibiting Diverse Properties. Biomed Reseach International, 19, 1-16.

Dang, N. H., Thanh, N. V., Kiem, P. V., Huong, L. M., Minh, C. V., Kim, Y. H. 2007. Two new triterpene glycosides from the Vietnamese sea cucumber Holothuria scabra. Archives of Pharmacal Research, 30, 1387-1391. 
Elyakov, G. B., Stonic, V. A., Levina, E. V., Slanke, V. P., Kuznetsova, T. A. 1973. Glycosides of marine invertebrates-I. A comparative study of the glycosides fraction of Pacific sea cucumbers. Comparative Biochemistry and Physiology, 44, 325-336.

Elyakov, G. B., Kuznetsova, T. A., Stonic, V. A., Levin, V. S., Albores, R. 1975. Glycosides of marine invertebrates-IV. A comparative study of the glycosides from Cuban sublittoral holothurians. Comparative Biochemistry and Physiology, 52, 413-417.

Esmat, A. Y., Said, M. M., Soliman, A. A., ELmasry, K. S., Badiea, E. A. 2013. Bioactive compounds, antioxidant potential, and hepatoprotective activity of sea cucumber (Holothuria atra) against thioacetamide intoxication in rats. Nutrition, 29, 258-267.

Fawzya, Y., Januar, H., Susilowati, R., Chasanah, E. 2015. Chemical composition and fatty acid profile of some Indonesian sea cucumbers. Squalen Bulletin of Marine and Fisheries Postharvest and Biotechnology, 10, 27-34.

Grabley, S. and Sattler, I. 2003. Natural products for lead identification: nature is a valuable resource for proving tools. In: A, Hillisch., R, Hilgenfeld., eds. Modern Methods of Drug Discovery. Birkhäuser, Basel: Springer, 87-107.

Haefner, B. 2003. Drugs from the deep: marine natural products as drug candidates. Drug Discovery Today, 8 , 536-544.

Han, H., Yi, Y. H., Liu, B. S., Wang, X. H., Pan, M. $\mathrm{X}$. 2008. Leucospilotaside $\mathrm{C}$, a new sulfated triterpene glycoside from sea cucumber Holothuria leucospilota. Chinese Chemical Letters, 19, 14621464.

Han, H., Yi, Y., Xu, Q., La, M., Zhang, H. 2009. Two new cytotoxic triterpene glycosides from the sea cucumber Holothuria scabra. Planta Medica, $75,1608-1612$
Han, H., Li, 1., Yi, Y.-H., Wang, X.-H., Pan, M.-X. 2012. Triterpene glycosides from sea cucumber Holothuria scabra with cytotoxic activity. Chin. Herb. Med, 4, 183-188.

Honey-Escandón, M., Arreguín-Espinosa, R., Solís-Marín, F.A., Samyn, Y. 2015. Biological and taxonomic perspective of triterpenoid glycosides of sea cucumbers of the family Holothuriidae (Echinodermata, Holothuroidea). Comparative Biochemistry and Physiology Part B: Biochemistry and Molecular Biology, 180, 16-39.

\section{https://doi.org/10.1016/j.cbpb.2014.09.007 http://www.marinespecies.org/}

Hua, H., Yi, Y.-H., Li, L., Liu, B.-S., Pan, M.-X., Yan, B., Wang, X.-H. 2009. Triterpene glycosides from sea cucumber Holothuria leucospilota. Chinise Journal of Natural Medicines, 7, 346-350.

Hue, N., Montagnac, A., Pais, M., Serani, L., Laprevote, O. 2001. Structural elucidation of eighteen cerebrosides from Holothuria coronopertusa in a complex mixture by highenergy collsion-induced dissociation of $[\mathrm{M}+\mathrm{Li}]^{+}$ ions. European Journal of Mass Spectrometry, 7, 409-417.

Kitagwa, I., Nishino, T., Matsuno, T., Akutsu, H., Kyogoku, Y. 1978. Structure of holothurine B, a pharmacologically active triterpene-oligoglycoside from the sea cucumber Holothuria leucospilota Brandt. Tetrahedron Letters, 11, 985-988.

Kitagwa, I., Nishino, T., Kyogoku, Y. 1979. Structure of holothurine A, a biologically active triterpene-oligoglycoside from the sea cucumber. Holothuria leucospilota Brandt. Tetrahedron Letters, 20, 1419-1422.

Kitagawa, I., Kobayashi, M., Son, B.W., Siuzukia, S., Kyogokub, Y. 1989. Marine natural products, XIX pervicosides A, B, and C, lanostanetype triterpene-oligoglycoside sulfates from the sea cucumber Holothuria pervicax. Chemical and pharmaceutical bulletin, 37, 1230-1234. 
Kobayashi, M., Hori, M., Kan, K., Yasuzawa, T., Matsui, M., Suzuki, S., Kitagawa, I. 1991. Marine natural product .XXVII. Distribution of lanostanetype triterpene oligoglycosides in ten kinds of Okinawan sea cucumbers. Chemical and pharmaceutical bulletin, 39, 2282-2287.

Lalli, C. M and Parson T. R. 1993. Biological Oceanography. New York, ButterworthHeinemann. Elsevier, 1-10.

Luo, L., Wu, M., Xu, L., Lian, W., Xiang, J., Lu, F., Gao, N., Xiao, C., Wang, S., Zhao, J. 2013. Comparison of physicochemical characteristics and anticoagulant activities of polysaccharides from three sea cucumbers. Marine Drugs, 11, 399-417.

Mansour, M. B., Balti, R., Yacoubi, L., Ollivier, V., Chaubet, F., Maaroufi, R. M. 2019. Primary structure and anticoagulant activity of fucoidan from the sea cucumber Holothuria polii. International Journal of Biological. Macromolecules, 121, 1145-1153.

Mercedes, G. W., Roggatz, C. C., Rodrigues, M. J., Barreira, L., Silva, M. M., Custódio, L. 2018. A new insight into the influence of habitat on the biochemical properties of three commercial sea cucumber species. International Aquatic Research, $10,361-373$.

Mohammed, I. A. 2016. Separation of a novel species from Actinopyga mauritiana (Holothuroidea: Holothuriidae) species complex, based on ecological, morphological and mitochondrial DNA evidence. Egypt. Egyptian Journal of Aquatic Biology and Fisheries, 20, 3945 .

Mondol, M. A., Shin, H. J., Rahman, M. A., Islam, M. T. 2017. Sea cucumber glycosides: Chemical structures, producing species, and important biological properties. Marine Drugs, 15, 1-35.

Nobsathian, S., Tuchinda, P., Sobhon , P., Tinikul, Y., Poljaroen, J., Tinikul, R., Sroyraya, M., Poomton, T., Chaichotranunt, S. 2017. An antioxidant activity of the whole body of Holothuria scabra. Chemical and Biological Technologies in Agriculture, 4, 1-5.

O'loughlin, P. M., Paulay, G., Vandenspiegel, D., Samyn, Y. 2007. New Holothuria species from
Australia (Echinodermata: Holothuroidea: Holothuriidae), with comments on the origin of deep and cool holothuriids. Memoirs of Museum Victoria, 64, 35-52.

Ridwanudin, A., Indriana, L., Kunzmann, A. 2018. No difference in nutritional profiles of wild and cultured juvenile sandfish, Holothuria scabra. Annual Research \& Review in Biology, 26, 1-11.

Ridzwan, B.H., Hanita, M.H., Nurzafirah, M., Siti Norshuhadaa, M. P., Farah Hanis, Z. 2014. Free Fatty Acids Composition in Lipid Extracts of Several Sea Cucumbers Species from Malaysia. International Journal of Bioscience, Biochemistry and Bioinformatics, 4, 204-207.

Rodriguez, J., Castro, R., Riguera, R. 1991. Holothurinosides: New antitumour non sulphated triterpenoid glycosides from the sea cucumber Holothuria forskalii, Tetrahedron, 47, 4753-4762.

Santos, R., Dias, S., Tecelão, C., Pedrosa, R., Pombo, A. 2017. Reproductive biological characteristics and fatty acid profile of Holothuria mammata. SPC Beche-de-mer Information Bulletin, 37, 57-64.

Silchenko, A. S., Stonik, V. A., Avilon, S. A., Kalinin, V. I., Kalinovsky, A. I., Zaharenko, A. M., Smirnov, A. V., Mollo, E., Cimino, G. 2005. Holothurins $\mathrm{B}_{2}, \quad \mathrm{~B}_{3}$ and $\mathrm{B}_{4}$, new triterpene glycosides from Mediterranean sea cucumbers of the genus Holothuria. Journal of Natural Products, $68,564-567$.

Sroyraya, M., Hanna, P. J., Siangcham, T., Tinikul, R., Jattujan, P., Poomtong, T., Sobhon, P. 2017. Nutritional components of the sea cucumber Holothuria scabra. Functional Foods in Health and Disease, 7, 168-181.

Stonik, V.A. 1986. Some terpenoid and steroid derivatives from echinoderms and sponges. Pure and Applied Chemistry, 58, 423-436.

Sun, G.-Q., Li, L., Yi, Y.-H., Yuan, W.-H., Liu, B.S., Weng, Y.-Y., Zhang, S.-L., Sun, P., Wang, Z.L. 2008. Two new cytotoxic nonsulfated pentasaccharide holostane $(=20$-hydroxylanostan18-oic acid gamma-lactone) glycosides from the sea cucumber Holothuria grisea. Helvetica Chimica Acta, 9, 1453-1460. 
Wen, J., Hu, C., Fan, S. 2010. Chemical composition and nutritional quality of sea cucumbers. Journal of the Science of Food and Agriculture, 90, 2469-2474.

Wu, J., Yi, Y. H., Tang, H. F., Wu, H. M., Zou, Z. R., Lin, H. W. 2006a. Nobilisides A - C, Three New Triterpene Glycosides from the Sea Cucumber Holothuria nobilis. Planta Medica, 72, 932-935.

Wu, J., Yi, Y. H., Tang, H.F., Zou, Z. R.,Wu, H. M. 2006b. Structure and cytotoxicity of a new lanostane-type triterpene glycoside from the sea cucumber Holothuria hilla. Chemistry \& Biodiversity, 3, 1249-1254.

Wu, J., Yi, Y. H., Tang, H. F.,Wu, H. M., Zhou, Z. R. 2007. Hillasides A and B, two new cytotoxic triterpene glycosides from the sea cucumber Holothuria hilla Lesson. Journal of Asian Natural Products Research, 9, 609-615.

Wu, M., Xu, L., Zhao, L., Xiao, C., Geo, N., Luo, L., Yang, L., Li, Z., Chen, L., Zhao, J. 2015. Structural analysis and anticoagulant Activities of the novel sulfated fucan possessing a regular welldefined repeating unit from sea cucumber. Marine Drugs, 13, 2063-2084.

Yahyavi, M., Afkhami, M., Javadi, A., Ehsanpour, M., Khazaali, A., Koshnood, R., Mokhlesi, A. 2012. Fatty acid composition in two sea cucumber species Holothuria scabra and Holothuria leucospilata from Qeshm island (Persian Gulf). African Journal of Biotechnology, 11, 2862-2868.

Yamada, K., Harada, Y., Nagaregawa, Y., Miyamoto, T., Isobe, R., Higuchi, R. 1998. Isolation and structure of biologically active gangliosides from the sea cucumber Holothuria pervicax. Journal of Organic Chemistry, 48, 2519-2525.

Yamada, K., Harada, Y., Miyamoto, T., Isobe, R., Higuchi, R. 2000. Constituents of Holothuroidea.9. Isolation and structure of a new ganglioside molecular species from the sea cucumber
Holothuria pervicax. Chemical and Pharmaceutical Bulletin, 48, 157-159.

Yamada, K., Matsubara, R., Kaneko, M., Miyamoto, T., Higuchi, R. 2001. Isolation and structure of a biologically active ganglioside molecular species from the sea cucumber Holothuria leucospilota. Chemical and Pharmaceutical Bulletin, 49, 447-452.

Yamada, K., Sasaki, K., Harada, Y., Isobe, R., Higuchi, R. 2002. Isolation and structure of glucocerebrosides from the sea cucumber Holothuria pervicax. Chemical and Pharmaceutical Bulletin, 50, 1467-1470.

Yamada, K., Onaka, H., Tanaka, M., Inaki, M., Higuchi, R. 2005. Determination of absolute configuration of the branched methyl group in anteiso type side chain moiety on long chain base of glucocerebroside from the sea cucumber Holothuria leucospilota. Chemical and Pharmaceutical Bulletin, 53, 1333-1334.

Yasumoto, T., Nakamura, K., Hashimoto, Y. 1967. A new saponin holothurin B isolated from the sea cucumber Holothuria vagabunda and Holothuria lubrica. Agricultural and Biological Chemistry, 31, 7-10.

Yuan,W.H., Yi, Y. H., Tan, R. X.,Wang, Z. L., Sun, G. Q., Xue, M., Zhang, H. W., Tang, H. F. 2009. Antifungal triterpene glycosides from the sea cucumber Holothuria (Microthele) axiloga. Planta Medica, 75,647-653.

Zhang, S., Yi, Y. H., Tang, H. F. 2006. Bioactive triterpene glycosides from the sea cucumber Holothuria fuscocinerea. Journal of Natural Products, 69, 1492-1495.

Zhang, J. J., Dai, J. B., Chen, L. L., Ding, P. Y., WU, J. 2008. Chemical constituents from sea cucumber Holothuria nobilis. Journal of Chinese medicinal materials, 31, 538-539. 